\title{
Active Luminous Blue Variables in the Large Magellanic Cloud
}

\author{
Nolan R. Walborn ${ }^{1,6}$, Roberto C. Gamen ${ }^{2,7}$, Nidia I. Morrell ${ }^{3}$, Rodolfo H. Barbá ${ }^{4,8,9}$, \\ Eduardo Fernández Lajús ${ }^{2,7}$, and Rodolfo Angeloni ${ }^{5}$ \\ ${ }^{1}$ Space Telescope Science Institute, 3700 San Martin Drive, Baltimore, MD 21218, USA; walborn@stsci.edu \\ ${ }^{2}$ Instituto de Astrofísica de La Plata, CONICET-UNLP and Facultad de Ciencias Astronómicas y Geofísicas, UNLP, \\ Paseo del Bosque s/n, La Plata, Argentina; rgamen@fcaglp.unlp.edu.ar, eflajus@fcaglp.unlp.edu.ar \\ ${ }^{3}$ Las Campanas Observatory, Carnegie Observatories, Casilla 601, La Serena, Chile; nmorrell@ 1co.cl \\ ${ }^{4}$ Departamento de Física y Astronomía, Universidad de La Serena, Cisternas 1200 Norte, La Serena, Chile; rbarba@dfuls.cl \\ ${ }^{5}$ Gemini Observatory, Colina El Pino, Casilla 603, La Serena, Chile; rangelon@gemini.edu \\ Received 2016 October 17; revised 2017 February 15; accepted 2017 February 18; published 2017 June 20
}

\begin{abstract}
We present extensive spectroscopic and photometric monitoring of two famous and currently highly active luminous blue variables (LBVs) in the Large Magellanic Cloud (LMC), together with more limited coverage of three further, lesser known members of the class. R127 was discovered as an Ofpe/WN9 star in the 1970s but entered a classical LBV outburst in or about 1980 that is still in progress, thus enlightening us about the minimum state of such objects. $\mathrm{R} 71$ is currently the most luminous star in the LMC and continues to provide surprises, such as the appearance of [Ca II] emission lines, as its spectral type becomes unprecedentedly late. Most recently, R71 has developed inverse P Cyg profiles in many metal lines. The other objects are as follows: HDE 269582, now a "second R127" that has been followed from Ofpe/WN9 to A type in its current outburst; HDE 269216, which changed from late B in 2014 to AF in 2016, its first observed outburst; and R143 in the 30 Doradus outskirts. The light curves and spectroscopic transformations are correlated in remarkable detail and their extreme reproducibility is emphasized, both for a given object and among all of them. It is now believed that some LBVs proceed directly to core collapse. One of these unstable LMC objects may thus oblige in the near future, teaching us even more about the final stages of massive stellar evolution.
\end{abstract}

Key words: Magellanic Clouds - stars: early-type - stars: massive - stars: peculiar - stars: variables: S Doradus supergiants

\section{Introduction}

Luminous blue variables (LBVs) signal a spectacular transition phase in massive stellar evolution and have attracted attention since the beginnings of astronomical observations, yet their progenitors, physics, and immediate fates remain unclear. Their impressive, recurrent outburst episodes, which produce major spectral and visual luminosity variations, as well as the less frequent giant eruptions that create circumstellar nebulae, have often been described for examples in the Milky Way and nearby galaxies, but a definitive identification of the physical mechanisms underlying these phenomena remains elusive. Even the phenomenology itself is incompletely known, with new behaviors recently being discovered, e.g., the appearance of far red [Ca II] lines in Radcliffe (R)71 (Feast et al. 1960) of the Large Magellanic Cloud (LMC; Gamen et al. 2012b; Walborn et al. 2012; Mehner et al. 2013). One difficulty is that the timescales of these events often exceed the careers, or at least the attention spans, of individual investigators. For instance, the end of a three-decade outburst of R127 (also in the LMC) was announced by Walborn et al. (2008), only to have it resume before the paper appeared and continue to this day!

\footnotetext{
${ }^{6}$ Staff Astronomer, STScI, which is operated by AURA, Inc. under NASA contract NAS5-26555.

7 Visiting Astronomer, Complejo Astronómico El Leoncito, operated under agreement between the Consejo Nacional de Investigaciones Científicas y Técnicas de la República Argentina and the National Universities of La Plata, Córdoba, and San Juan.

8 Visiting Astronomer, Las Campanas Observatory, Carnegie Observatories.

${ }^{9}$ Visiting Astronomer, European Southern Observatory, La Silla.
}

Another challenge is that the outbursts are sudden and unpredictable. Thus, continuous, at least monthly monitoring is essential in order not to miss something important. Until recently, it was assumed that the LBV phase would be followed by a Wolf-Rayet phase, but recent evidence suggests that some LBVs may proceed directly to core collapse (e.g., Kotak \& Vink 2006; Groh et al. 2013; Mauerhan et al. 2013a).

At visual maximum, these objects become the brightest stars in the LMC, exceeding 9th mag in the most extreme cases. As is well known, the bolometric magnitudes change little or not at all during their outbursts, with the ultraviolet flux being reprocessed progressively to longer wavelengths as the temperature of the expanding false photosphere decreases and the apparent spectral type becomes later. The outbursts subsequently invert and then recur. Clearly, improved physical understanding of the LBV phenomena is essential for a complete view of the end states of massive stars.

To that end, here we present our spectroscopic and photometric monitoring since 2008 of five LBVs in the LMC, together with their historical light curves as available. These data reveal a number of new and surprising features; however, some gaps in the records create uncertainties and it is essential to monitor these objects continuously. The reproducibility of the correlated spectral and light variations both for a given object and among different objects is remarkable and will be emphasized throughout. The details of our observations are given in Section 2, and the results for each object are described in turn in Section 3. Finally, some global inferences and questions are discussed in Section 4. 


\section{Observations}

Identifications of our five targets are listed in Table 1, along with the archetypical LMC LBV S Doradus for which we do not provide new data but which is included in the Discussion below. We emphasize that all of these objects except for R71 are located in OB clusters or associations, as listed and referenced. The specifications of the seven spectrographs and four cameras we used for new observations are given in the following subsections. Some further spectroscopic data were obtained from the ESO archive, as noted in the tables.

\subsection{Low-resolution Spectroscopy}

\subsubsection{Las Campanas Observatory}

All of our targets have been observed with the Boller \& Chivens $(\mathrm{B} \& \mathrm{C})$ spectrograph attached to the $2.5 \mathrm{~m} \mathrm{du}$ Pont telescope at Las Campanas. We used the 1200 line $\mathrm{mm}^{-1}$ grating (blazed at $5000 \AA$ ) tilted at an angle of $18^{\circ}$ and a $150 \mu \mathrm{m}$ slit. This configuration provides spectral coverage from 3900 to $5500 \AA$ at a resolution of $\sim 1.7 \AA$ as measured from the FWHM of the HeNeAr comparison lines. Dome flats and bias frames were obtained in the afternoon previous to each observing night, and a set of spectrophotometric standards was used to perform flux calibration. The spectrograms were then normalized. All reductions were performed with IRAF routines.

R71 and R127 were observed with the $6.5 \mathrm{~m}$ Magellan I Baade telescope at Las Campanas on 2009 August 29. We used the Inamori Magellan Areal Camera and Spectrograph (IMACS) in its long-camera $(f / 4)$ mode, with a 600 line $\mathrm{mm}^{-1}$ grating and a 0 .' 9 long slit providing coverage from 3700 to $6800 \AA$ at a spectral resolution of $1.4 \AA$ as measured from the FWHM of the HeNeAr comparison lamp lines. The usual set of flats and bias was secured, as was a sample of spectrophotometric standards. The flux-calibrated data were then normalized. All reductions were performed with IRAF.

Spectrograms of all our targets but HDE 269216 have been obtained with the Magellan Echellette (MagE) on the $6.5 \mathrm{~m}$ Magellan telescopes at Las Campanas using a $1^{\prime \prime}$ slit. The resolving power is 4100 with full wavelength coverage in the optical. Wavelength calibration was performed with a ThAr lamp. A set of flux standards was also observed on each observing night. Reductions were performed with a combination of the mtools package, originally designed by Jack Baldwin for the reduction of Magellan MIKE data, and IRAF echelle tasks. For a more detailed description, see Massey et al. (2012).

One of our low-resolution spectrograms of HDE 269582 was obtained with the Wide Field Reimaging CCD Camera (WFCCD) on the $2.5 \mathrm{~m}$ du Pont telescope at Las Campanas in the context of an extensive survey of WNL stars in the Large Magellanic Cloud (Schnurr et al. 2008). We used the blue H\&K grism and a $150 \mu \mathrm{m}$ long slit yielding a wavelength coverage from 3800 to $5600 \AA$ at a resolution of $3.1 \AA$.

\subsection{High-resolution Spectroscopy}

\subsubsection{Las Campanas Observatory}

Most of the high-resolution spectrograms presented here were obtained with the echelle spectrograph available at the
$2.5 \mathrm{~m} \mathrm{du}$ Pont telescope on Las Campanas through a $1^{\prime \prime}$ slit, which provides a resolving power of $\sim 25,000$ and a wavelength coverage from 3500 to $9000 \AA$. Wavelength calibration was obtained with a ThAr lamp. Reductions were performed with the IRAF echelle package.

High-resolution spectrograms of R71, R127, and HDE 269582 were obtained with the $6.5 \mathrm{~m}$ Magellan II Clay telescope and the Magellan Inamori Kyocera Echelle (MIKE) spectrograph with the 0.7 slit and $2 \times 2$ binning on the detectors. This configuration provides a resolving power of $\sim 34,000$. Exposures of a ThAr lamp were used for wavelength calibration. MIKE data were reduced with the mools package (see MagE above).

\subsubsection{European Southern Observatory}

Several of our high-resolution spectrograms are from the ESO/ La Silla Fiber-Fed Extended Range Optical Spectrograph (FEROS; Kaufer et al. 1999), first at the $1.5 \mathrm{~m}$ and later at the $2.2 \mathrm{~m}$ telescope. The resolving power is 48,000 .

\subsection{Photometry}

\subsubsection{Las Campanas Observatory}

Broadband $V$ images of R71, R127, R143, and HDE 269582 were kindly obtained by the Carnegie Supernova Project (CSP) team with the $1 \mathrm{~m}$ Swope telescope at Las Campanas during the course of their campaigns. Starting in late 2013, the original SITe\#3 detector was replaced by a $4 \mathrm{~K}$ E2V chip. Observations and reductions are described in Contreras et al. (2010). The accuracy of these measurements is $\pm 0.05 \mathrm{mag}$.

\subsubsection{Complejo Astronómico El Leoncito}

Direct CCD images of R71 and R127 were obtained in the $B$, $V, R$, and $I$ bands between 2010 and 2016 by EFL with the $2.15 \mathrm{~m}$ "Jorge Sahade" telescope at the Complejo Astronómico El Leoncito (CASLEO), San Juan, Argentina.

A VersArray $1300 B$ Roper Scientific $(1340 \times 1300$ pix $)$ CCD camera attached to the modified Cassegrain focus of the telescope was used until early 2012. This camera was subsequently replaced by a VersArray 2048 B $(2048 \times 2048$ pix $)$ model.

The images were calibrated in the typical manner with flatfield and bias images in the CCDRED/IRAF package. Aperture photometry was finally performed using the APPHOT/IRAF package. The accuracy of these measures is $\pm 0.05 \mathrm{mag}$.

\subsubsection{Cerro Tololo Inter-American Observatory}

BVRI images of R71, R127, R143, and HDE 269582 were obtained by RA with the SMARTS Consortium $1.3 \mathrm{~m}$ telescope at the Cerro Tololo Inter-American Observatory (CTIO), Chile, over semesters 2012B-2014A. The observations were conducted on a weekly basis with the ANDICAM dual-channel imager. The optical detector is a Fairchild 447 $2048 \times 2048$ pix CCD offering a $\sim 6^{\prime} \times 6^{\prime}$ field of view with a scale of 0 " $371 \mathrm{pix}^{-1}$ in $2 \times 2$ binning. The optical data are delivered by the SMARTS Consortium bias subtracted and flat-field calibrated. The entire reduction pipeline is described on the SMARTS Github page. ${ }^{10}$

\footnotetext{
${ }^{10}$ https://github.com/SMARTSconsortium/ANDICAM
} 
Table 1

Identification of the LBVs

\begin{tabular}{|c|c|c|c|c|c|c|c|}
\hline \multicolumn{4}{|c|}{ Stellar Designations } & \multicolumn{2}{|c|}{ Coordinates } & \multirow{2}{*}{ Cluster/Assoc. ${ }^{\mathrm{a}}$} & \multirow{2}{*}{$A_{V}^{\mathrm{b}}$} \\
\hline Name & $R$ & $\mathrm{HD} / \mathrm{HDE}$ & $\mathrm{Sk}$ & $\alpha_{2000}$ & $\delta_{2000}$ & & \\
\hline$\ldots$ & 71 & 269006 & $-71^{\circ} 3$ & 05:02:07.392 & $-71: 20: 13.12$ & none & $0.15,0.37,0.63$ \\
\hline$\ldots$ & $\ldots$ & 269582 & $-69^{\circ} 142 \mathrm{a}$ & $05: 27: 52.657$ & $-68: 59: 08.56$ & NGC 1983 & 0.72 \\
\hline$\ldots$ & 127 & 269858 & $-69^{\circ} 220$ & 05:36:43.688 & $-69: 29: 47.52$ & NGC 2055 & 0.60 \\
\hline$\cdots$ & $143^{\mathrm{c}}$ & $\ldots$ & $\ldots$ & $05: 38: 51.623$ & $-69: 08: 07.19$ & R143/30 Dor & 2.0 \\
\hline
\end{tabular}

Notes.

${ }^{a}$ HDE 269216: Shapley \& Lindsay (1963), Schild (1987), Prinja \& Schild (1991); S Dor: Leitherer et al. (1985), Walborn (1991), Massey et al. (2014); HDE 269582: Hodge \& Wright (1967); R127: Heydari-Malayeri et al. (2003); R143: Walborn \& Blades (1997).

${ }^{\mathrm{b}} \mathrm{R}=3$ assumed. R71: Mehner et al. (2013); HDE 269216: Prinja \& Schild (1991); S Dor: Massey (2000); HDE 269582: Shore \& Sanduleak (1984); R127: HeydariMalayeri et al. (2003): "Taking $(b-y)_{0}=-0.14 \mathrm{mag}$ as the average value and using the conversion relation $E(b-y)=0.67 \times E(B-V)+0.02($ Kaltcheva $\&$ Georgiev (1992)), we find $E(B-V)=0.18 \mathrm{mag}\left(A_{V}=0.6 \mathrm{mag}\right)$ for the average reddening of clusters R127/R128."; R143: Parker (1993).

${ }^{c}$ Often erroneously identified as HDE 269929, e.g., in SIMBAD and other literature-but not by Feast et al. (1960). The position of this star is 05:39:37.84, -70:39:47.0, as valuably measured by Brian Skiff (http://vizier.cfa.harvard.edu/viz-bin/VizieR-3?-source=B/mk).

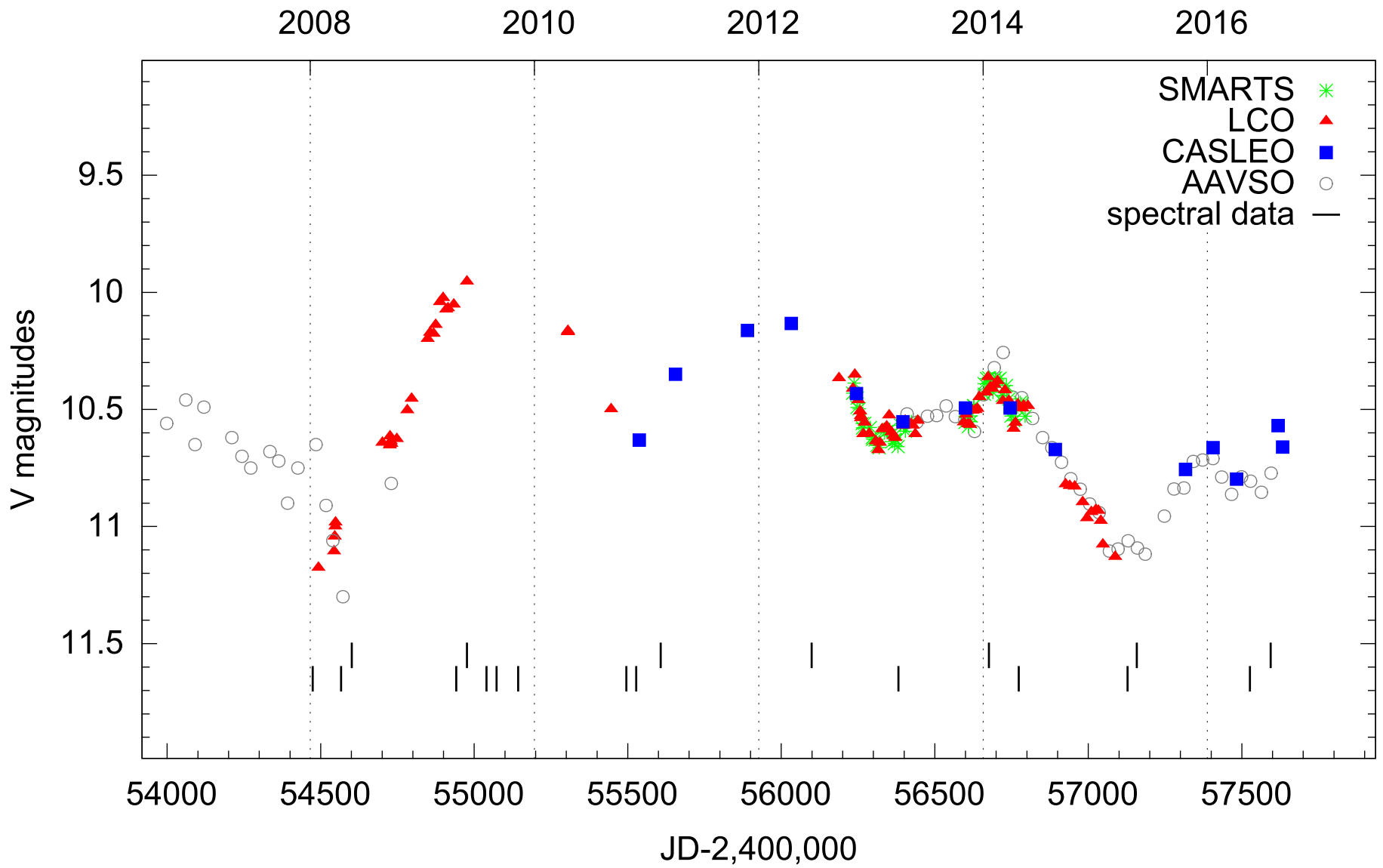

Figure 1. Recent/current visual light curve of R127. The AAVSO photometry through 2008 is visual, but as of 2013 it is CCD. SMARTS, LCO, and CASLEO data are discussed in Section 2.3. The spectroscopic epochs displayed in subsequent figures are marked by ticks at the bottom, with low resolution below high.

In 2017 January, new photometric observations were obtained by RHB with the Southeastern Association for Research in Astronomy/CTIO $0.61 \mathrm{~m}$ telescope (Mack et al. 2010) remotely from his home in La Serena. The result for R71 has been added here for comparison with striking spectroscopic developments.

\section{Results}

\section{1. $R 127$}

We pick up the story of this remarkable object where it was left by Walborn et al. (2008). The light curve is updated in Figure 1, which shows how the decades-long outburst that had 


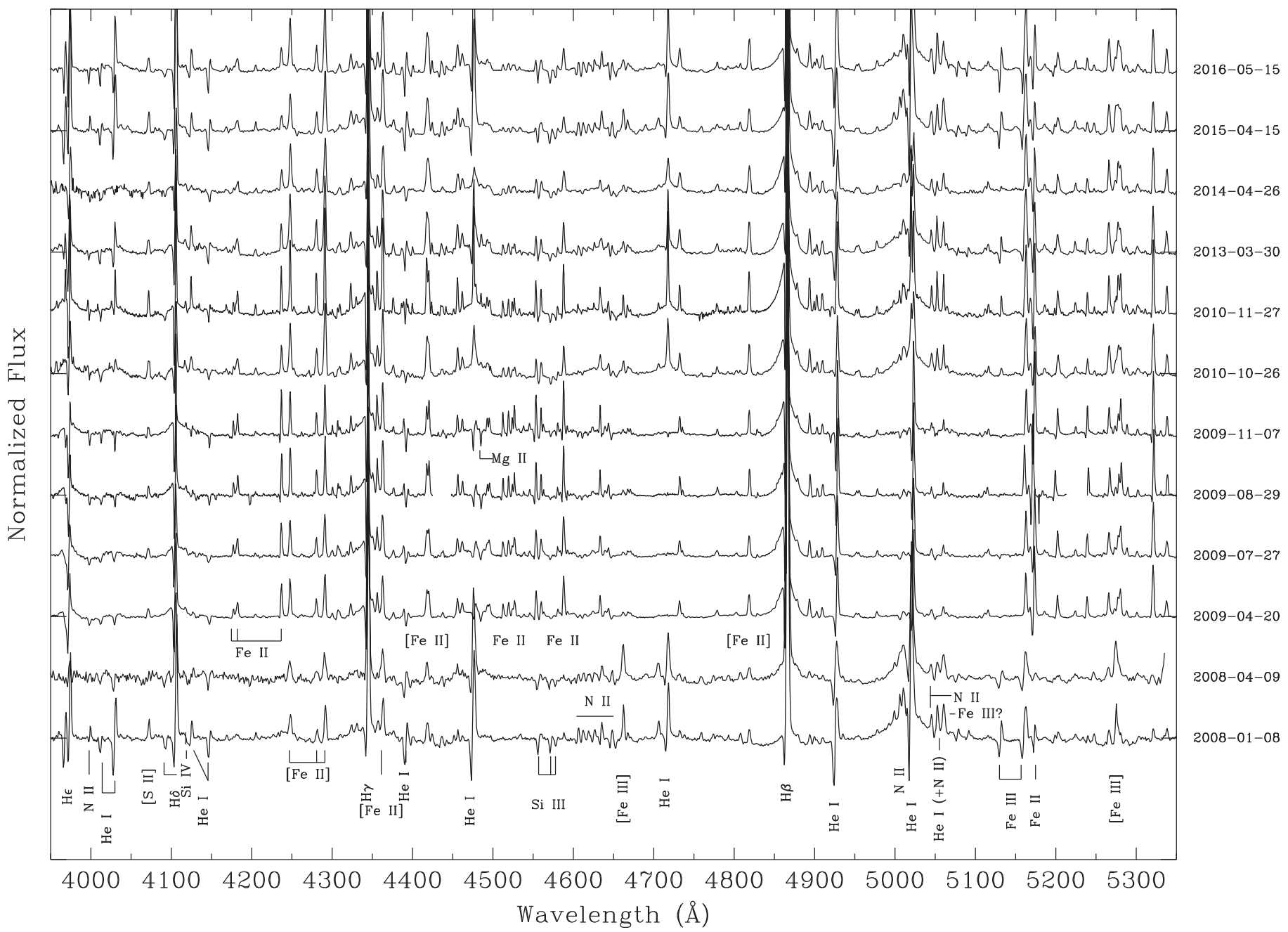

Figure 2. Low-resolution violet through green spectroscopy of R127, 2008-2016. In this and subsquent spectroscopic figures, the wavelength scales include the LMC velocity. The rest wavelengths of the marked features can be found in Walborn \& Fitzpatrick $(1990,2000)$ and Walborn \& Howarth (2000). He I $\lambda \lambda 4922,5015$ at hot phases are replaced by Fe II $\lambda \lambda 4924,5018$ at cooler ones. The ordinate ticks (and here the spectrograms) are separated by 0.50 continuum flux units.

Table 2

Low-resolution Spectroscopic Observations of R127

\begin{tabular}{lccl}
\hline \hline Observation Date & HJD-2450000.0 & Instrument & PI/Observer \\
\hline 2008 Jan 08 & 4473.6 & B\&C & N. Morrell \\
2008 Apr 09 & 4556.5 & R-C & R. Humphreys \\
2009 Apr 20 & 4941.5 & B\&C & R. Barbá \\
2009 Jul 27 & 5039.9 & B\&C & N. Morrell \\
2009 Aug 29 & 5072.8 & IMACS & N. Morrell \\
2009 Nov 07 & 5142.8 & MagE & P. Massey/N. Morrell \\
2010 Oct 26 & 5495.6 & B\&C & N. Morrell \\
2010 Nov 27 & 5527.8 & MagE & P. Massey/N. Morrell \\
2013 Mar 30 & 6381.5 & B\&C & N. Morrell \\
2014 Apr 26 & 6773.5 & B\&C & N. Morrell \\
2015 Apr 15 & 7127.5 & B\&C & N. Morrell \\
2016 May 17 & 7526.5 & B\&C & N. Morrell \\
\hline
\end{tabular}

Note.

${ }^{a}$ Cerro Tololo Inter-American Observatory $4 \mathrm{~m}$ Ritchey-Chretien Spectrograph.

begun in or about 1980, peaked around 1990, and declined more gradually (albeit with some fluctuations) until 2008, abruptly reversed into a steep rise that year. Thereafter, the
Table 3

High-resolution Spectroscopic Observations of R127

\begin{tabular}{lcll}
\hline \hline Observation Date & $\begin{array}{c}\text { HJD- } \\
2450000.0\end{array}$ & Instrument & PI/Observer \\
\hline 2008 May 14 & 4601.4 & FEROS & $\begin{array}{c}\text { R. Barbá/N. } \\
\text { Morrell } \\
\text { 2009 May 24 }\end{array}$ \\
& 4976.4 & FEROS & $\begin{array}{l}\text { R. Barbá/N. } \\
\text { Morrell }\end{array}$ \\
2011 Feb 14 & 5606.5 & FEROS & R. Barbá \\
2012 Jun 20 & 6098.9 & FEROS & R. Barbá \\
2014 Jan 01 & 6675.7 & MIKE & R. Barbá \\
2015 May 15 & 7158.5 & du Pont/echelle & N. Morrell \\
2016 Jul 24 & 7593.9 & du Pont/echelle & N. Morrell \\
\hline
\end{tabular}

irregular timescale of the reversals has decreased to 2-3 years with equally irregular amplitudes. Currently, the visual light is again rising for the fourth time since 2008. Our displayed spectroscopic epochs are marked. Further light curve information for R127 is presented by Szczygieł et al. (2010, their Figure 1). Clearly, the star is now subject to activity quite different from that of 1982-2008. Would that we knew how 

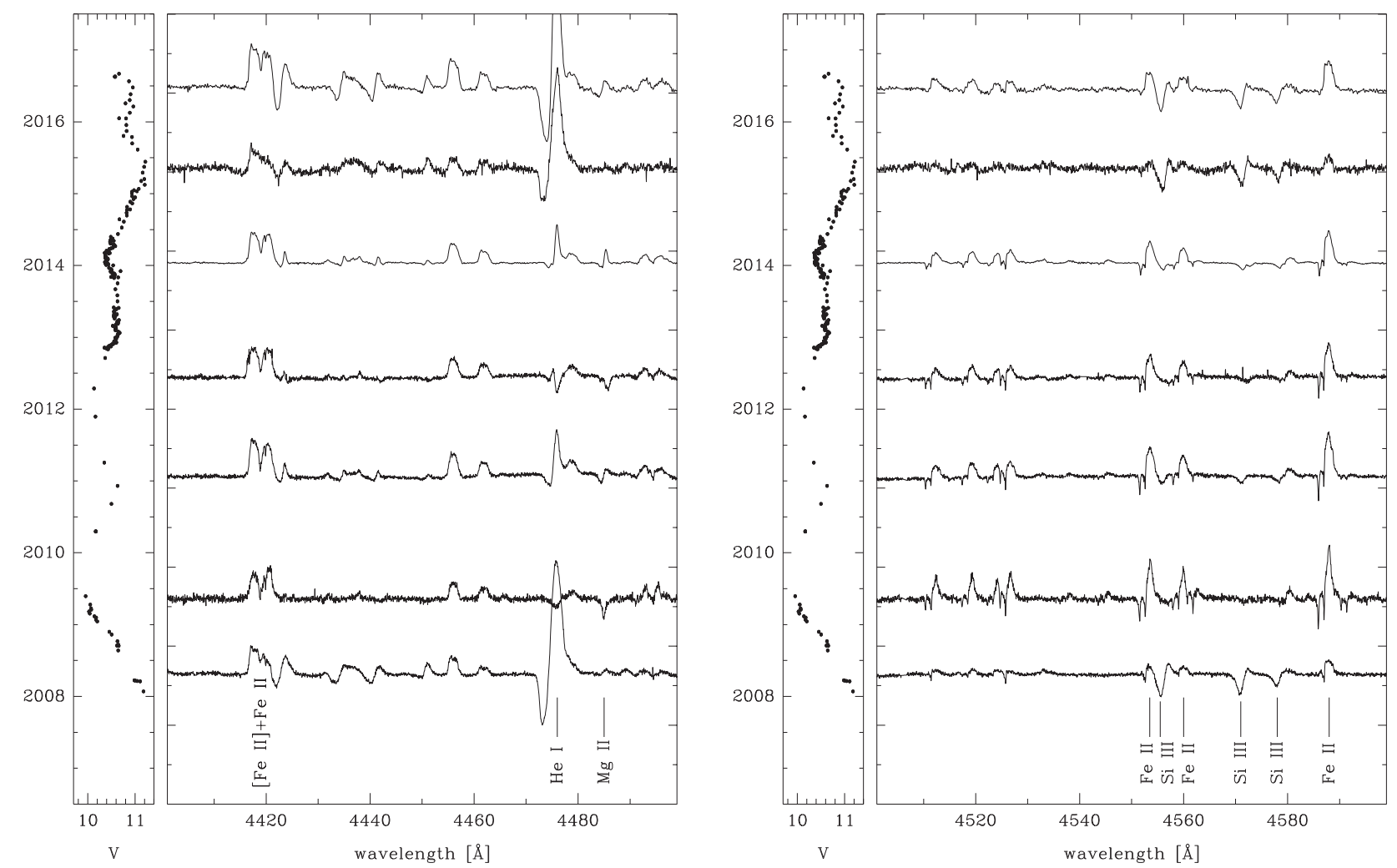

Figure 3. Segments of the high-resolution spectrograms of R127, 2008-2016: left, $4400 \AA$; right, $4500 \AA$. The light curve is displayed correlatively at the left of each panel. The spectral content corresponds to early-B type at the deep minima in 2008 and again in 2015-16, with weaker He I $\lambda 4471$ near the shallow minimum of 2011 and the similar shallow maximum of 2014, but predominantly A-type Fe II features at the strong 2009-10 and 2012 maxima (see line identifications in Walborn et al. 2008). The Si III $\lambda \lambda 4552-68-75$ triplet is prominent in absorption at the stronger B-type phases, with Fe II emission (e.g., $\lambda \lambda 4549,4556,4584)$ dominating at the A-type. (The heliocentric wavelength scales include a shift of $+4.4 \AA$ at $4500 \AA$ due to the radial velocity of R127.) The major ordinate ticks are separated by 1.0 continuum flux unit in these and all subsequent high-resolution montages unless otherwise indicated.
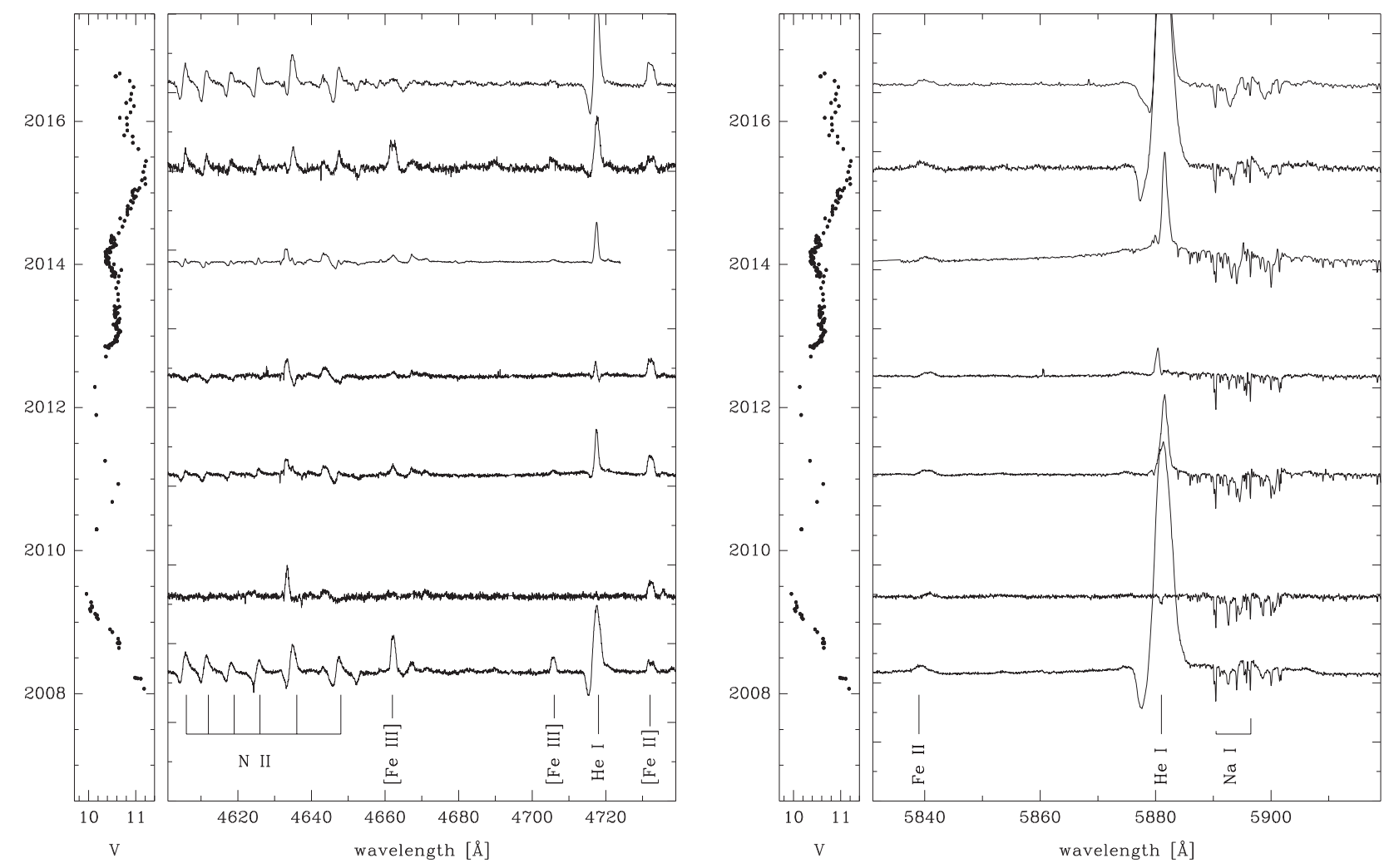

Figure 4. Same as Figure 3, but for left, 4600-4700 ̊; and right, 5800-5900 ̊. The N II multiplet at $\lambda \lambda 4601-07-14-21-31-43$ and He I $\lambda \lambda 4713$, 5876 are prominent in the B-type phases. The latter 2008, 2015, 2016 emission peaks reach 4.9, 5.3, 4.5 continuum units above zero. The Galactic interstellar Na I D doublet at $5890-96 \AA$ is marked, while intermediate-velocity and LMC doublets (including variable stellar components) occur at redshifted wavelengths. 

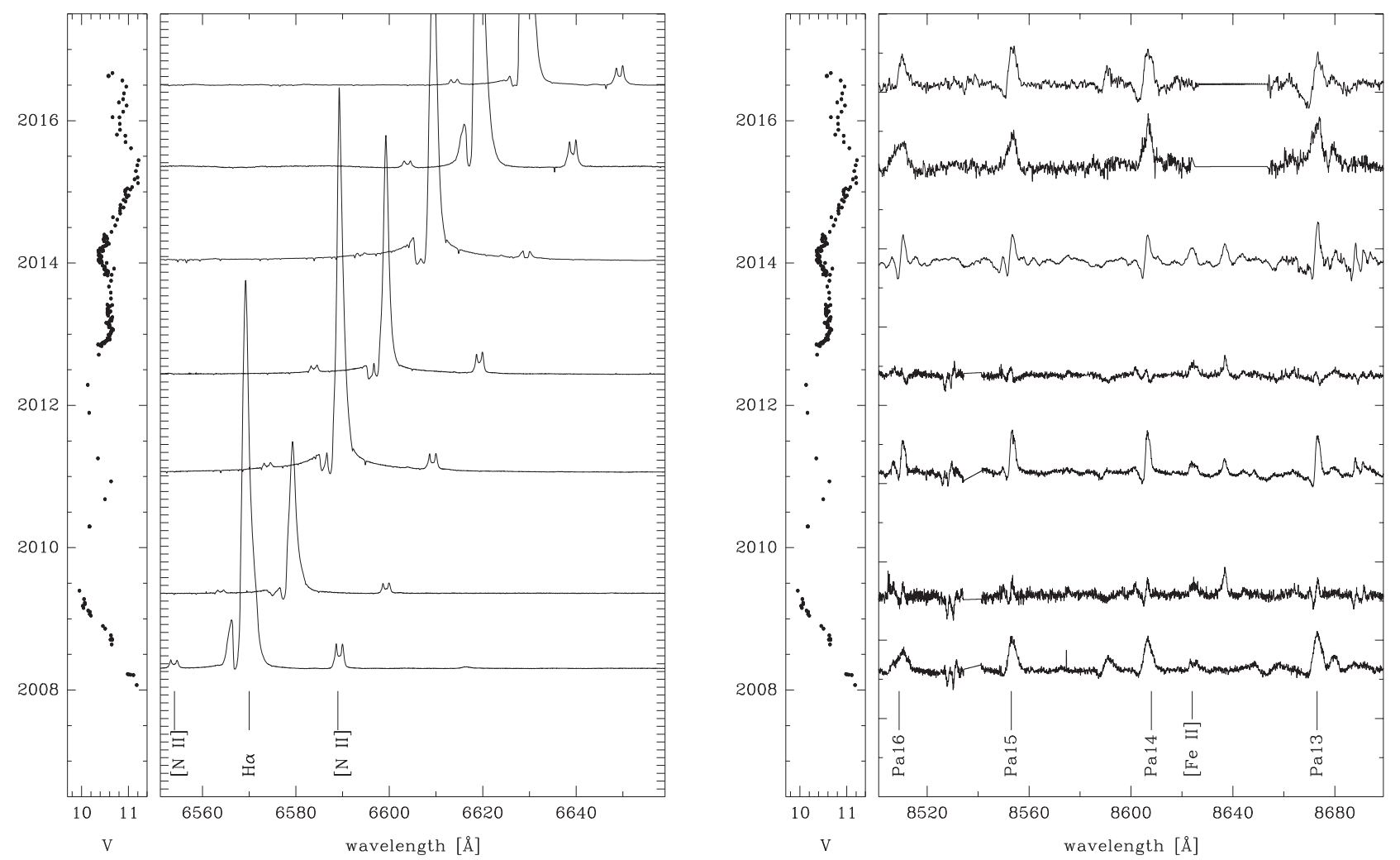

Figure 5. Same as Figure 3, but for left, $6500 \AA$; and right, $8500-8600 \AA$. The wavelength scale in the left panel corresponds to the bottom spectrogram only, while those above are arbitrarily and progressively shifted rightward to avoid superposition of the complex H $\alpha$ profiles. The emission peaks of 2014 and 2015 reach 43 and 41 continuum units above zero, respectively, while that of 2016 is saturated. The ordinate ticks in the left panel are still separated by 1.0 continuum unit, but the plotting scale is compressed by a factor of 10 . The double [N II] $\lambda \lambda 6548-83$ emission lines arise in the circumstellar nebula produced by a prior giant eruption (Walborn 1982). The right panel covers the Ca II Triplet $\lambda \lambda 8498-8542-8662$; however, the similar intensity and profile of Paschen (Pa) $14 \lambda 8598$ diagnoses a minimal presence of $\mathrm{Ca}$ II, so that the features near those wavelengths are also dominated by Paschen series members Pa16 $\lambda 8502, \mathrm{~Pa} 15 \lambda 8545$, and Pa13 $\lambda 8665$.

190019101920193019401950196019701980199020002010

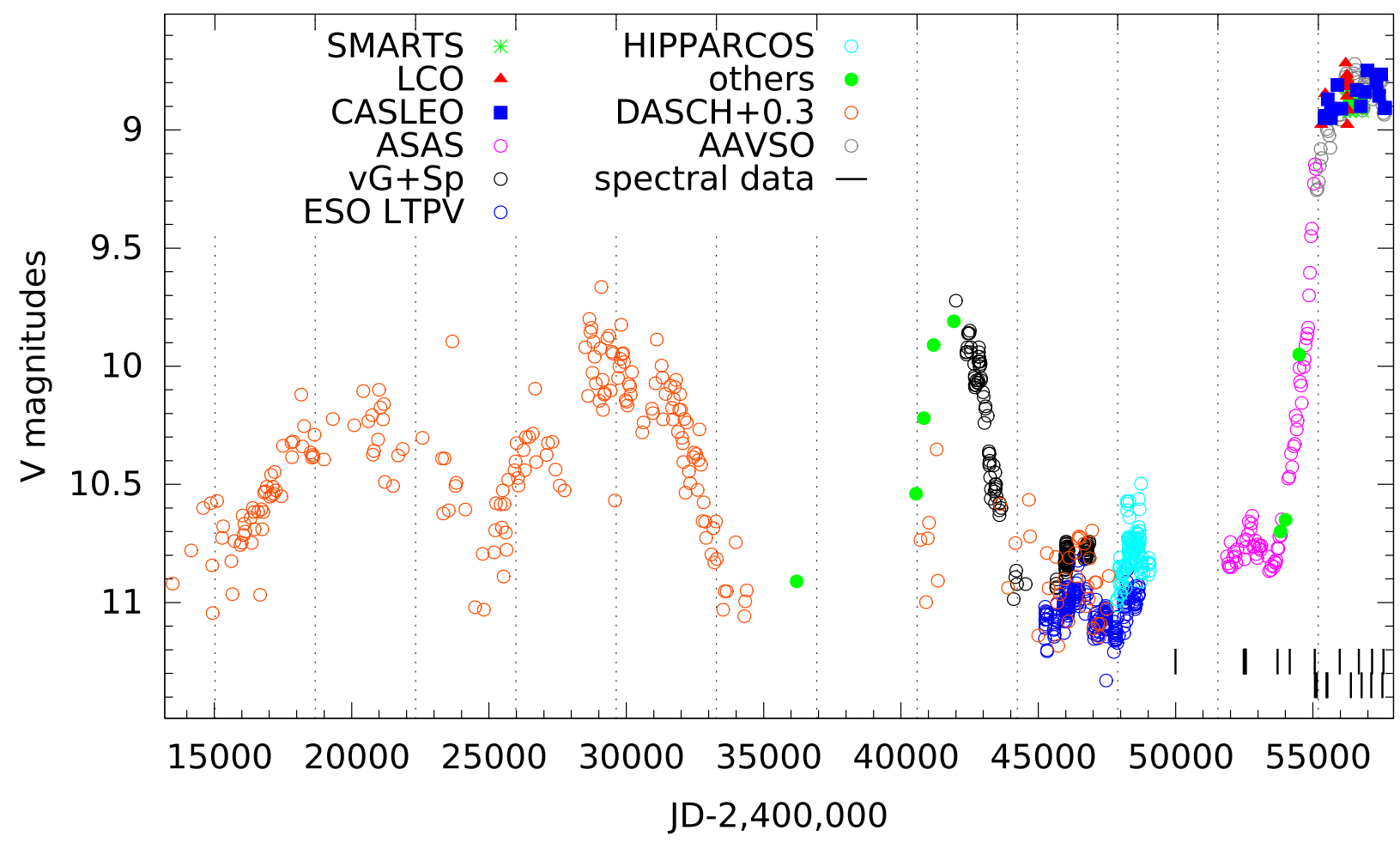

Figure 6. Historical visual light curve of R71. ASAS: Pojmanski (1997). vG+Sp: van Genderen (1979), Spoon et al. (1994). ESO: Manfroid et al. (1991). Hipparcos: ESA SP1200 (1997). DASCH: Grindlay et al. (2012), adjusted by +0.3 mag to match contemporaneous sources. Others: Mendoza (1970), Thackeray (1974), Munari et al. (2009). See the text for discussion. 
The Astronomical Journal, 154:15 (26pp), 2017 July

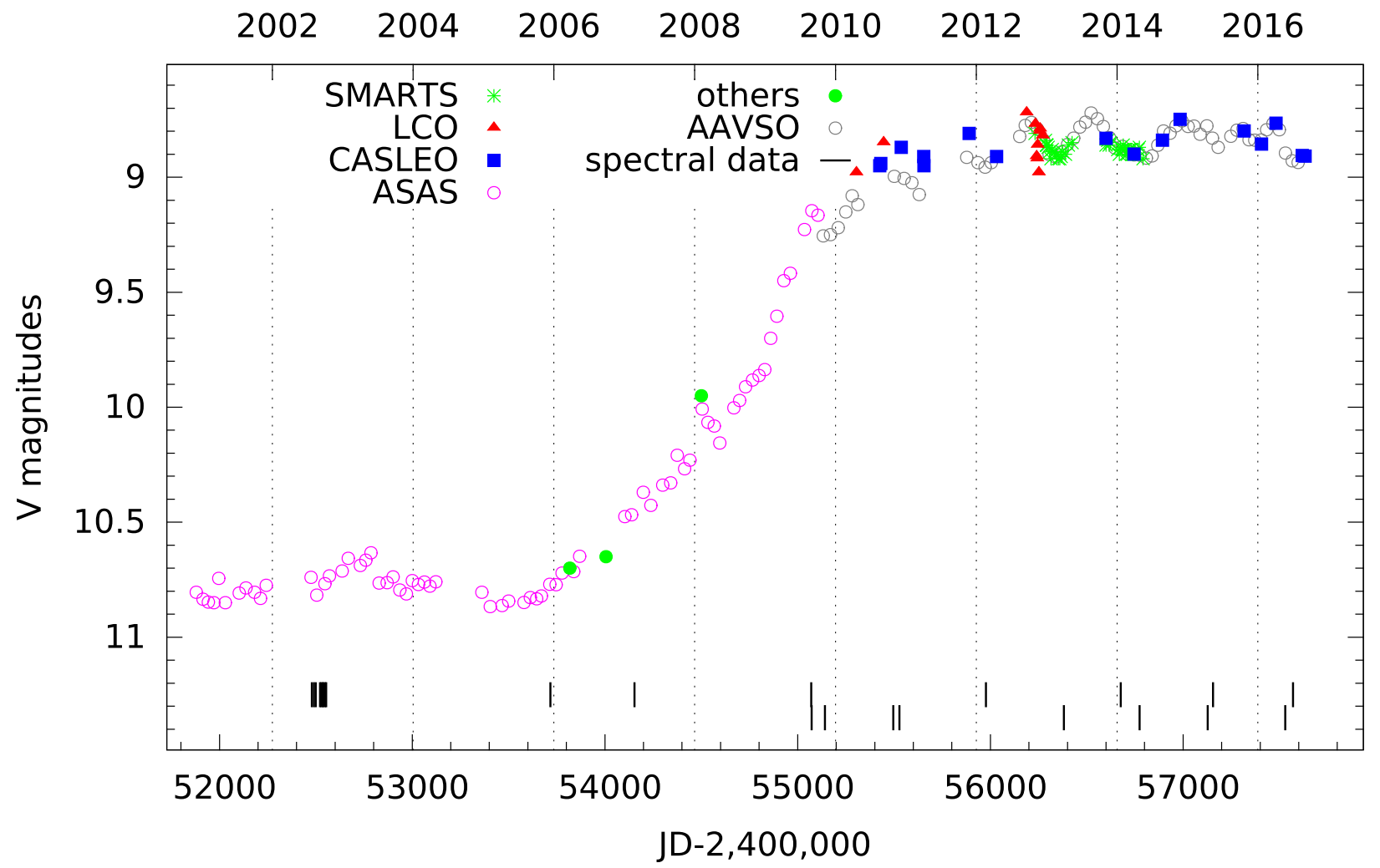

Figure 7. Expanded recent/current visual light curve of R71. Displayed spectroscopic epochs are marked at the bottom as in Figure 1. There is an appearance of a (pseudo?) periodicity throughout the supermaximum, which we have determined to be $445 \pm 40 \mathrm{~d}$ and which will be pursued in subsequent work.

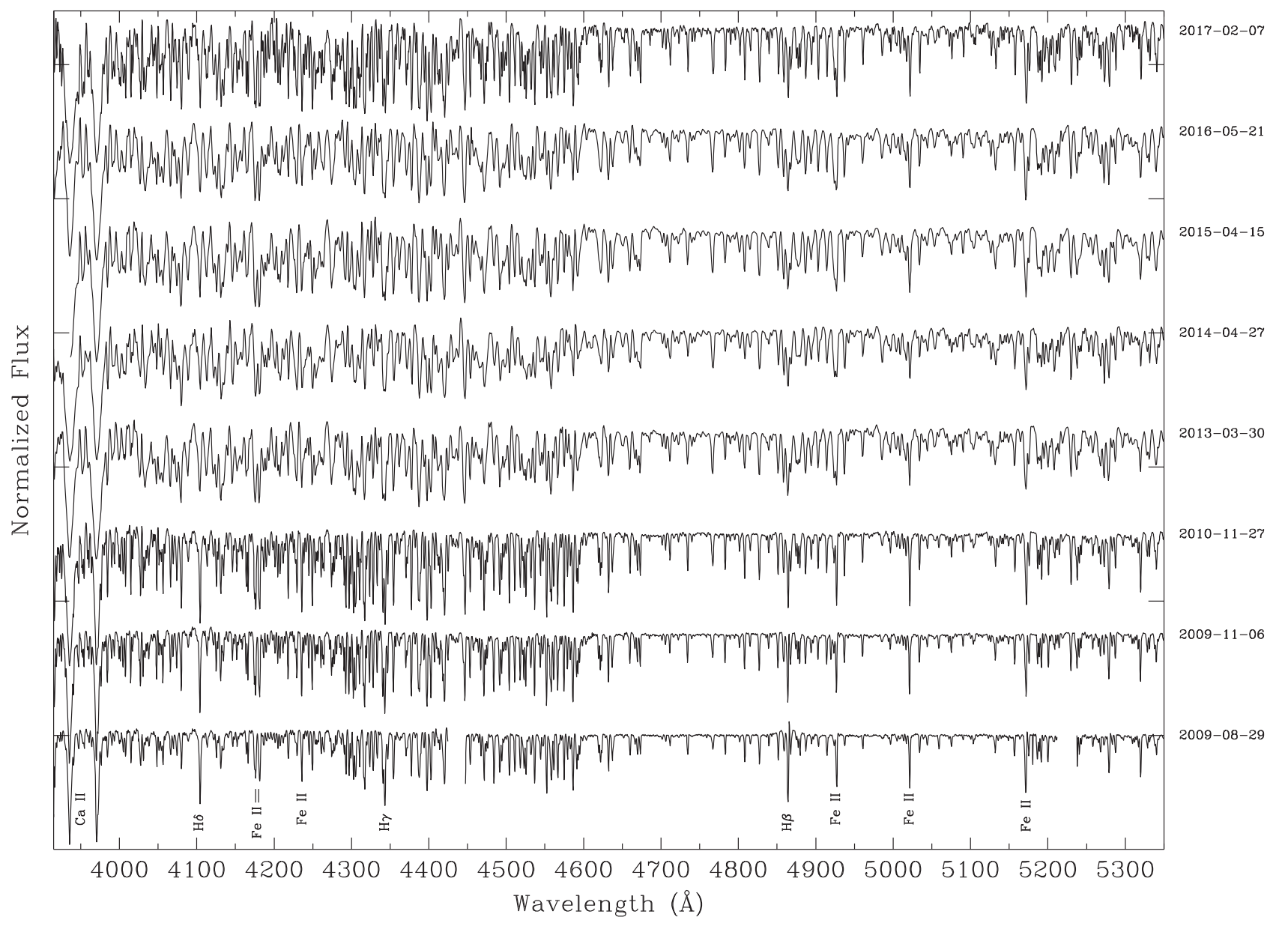

Figure 8. Low-resolution violet through green spectroscopy of R71, 2009-2017. The ordinate ticks are separated by 0.75 continuum units. See the text for discussion. 

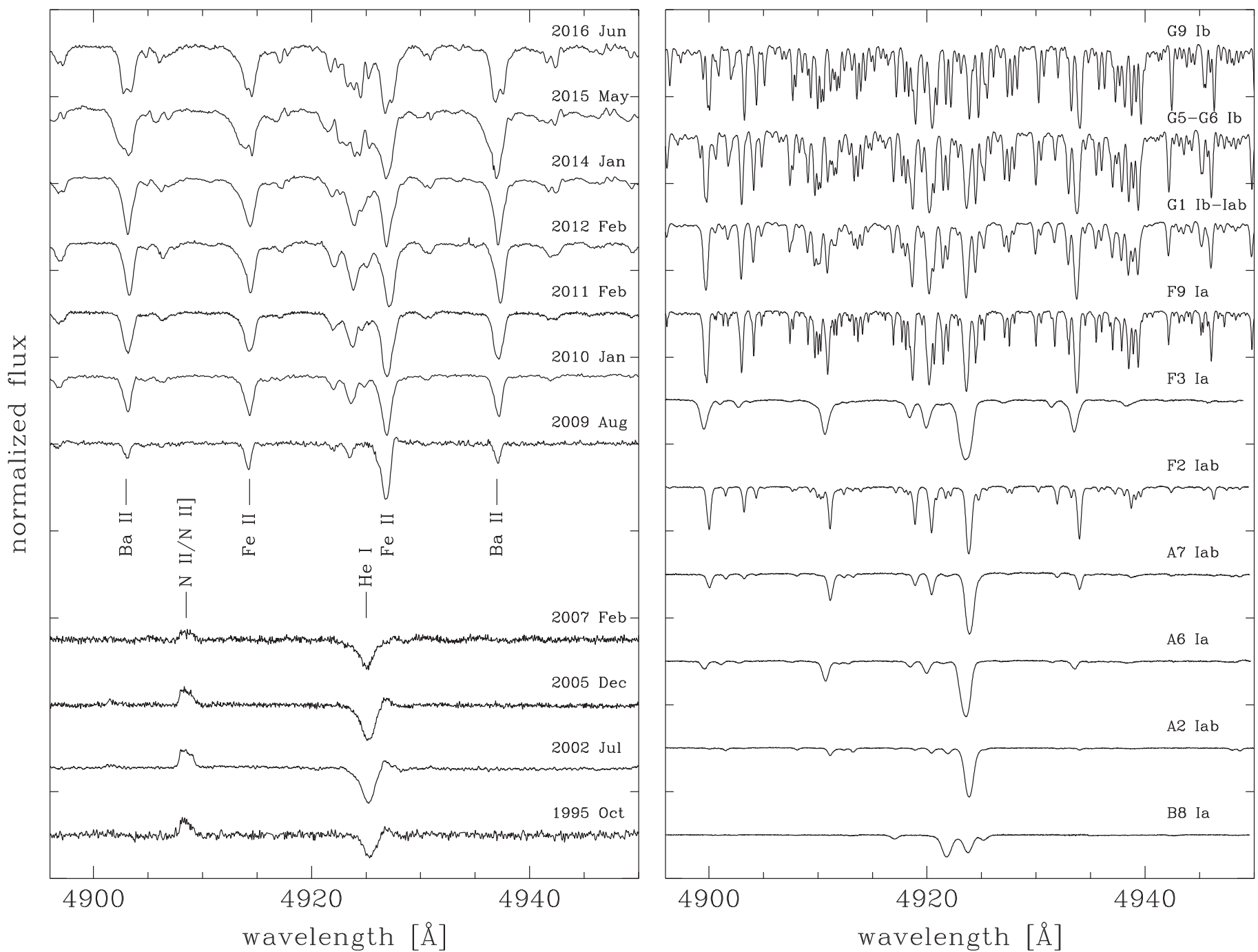

Figure 9. High-resolution $\lambda 4900$ spectroscopy of R71, 1995-2016, together with a UVES/POP supergiant sequence as classification standards. The Ba II $\lambda 4934$ and Fe II $\lambda 4924$ classification lines in the later types are marked at left, as is He I $\lambda 4922$ earlier. The ordinate ticks are separated by 1.0 continuum unit. See the text for details.

and why... Might it be "reverberating" and/or relaxing from the preceding "superoutburst?" Or, on the contrary, might it be approaching a final crisis? That has also been suggested for a distant hyperactive LBV by Pastorello et al. (2010). Again, continuing monitoring is essential.

The Ofpe/WN9 spectrum of R127 during the 1970s is reproduced by Walborn $(1977,1982)$. In Table 2 and Figure 2, we list and display all of our low-resolution spectroscopic data, providing complete coverage from violet through green beginning in 2008 through 2016. The first observation is of (peculiar) early-B type, but the salient features are already weakening three months later, and by the following year A-type features predominate. By the end of the sequence, this transformation is fully inverted. The changes tightly track the visual light curve (Figure 1), with earlier types corresponding to fainter states and later to brighter. Even minor light inflections are reflected in the spectra, as covered.

In Table 3 and Figures 3-5, we extend the high-resolution spectral sequences of Walborn et al. (2008) in both time and wavelength, focusing on highly variable individual features of particular interest. In each panel, the light curve is reproduced vertically on the left with the spectrograms placed at their corresponding epochs. Thus, the characteristic close correlations between the spectra and light can be optimally appreciated. Earlier types/features occur at minima (2008, 2011, 2013, 2015-16) and later types at maxima (2009, 2012, 2014). Even the degrees of the spectral transformations track the photometric amplitudes in striking detail. In particular, note the very strong He I P Cygni profiles at the deep 2008 and 2015-16 minima, which are absent at the shallower 2011 and 2013 ones. Further details about individual features are noted in the figure captions.

\section{2. $R 71$}

We begin the account of this likewise remarkable object with its historical light curve in Figure 6, recently extended retroactively to 1900 by means of the digitized Harvard Patrol data ("DASCH;" Grindlay et al. 2012; Walborn et al. 2014), which reveal two broad, contiguous outbursts between 1900 

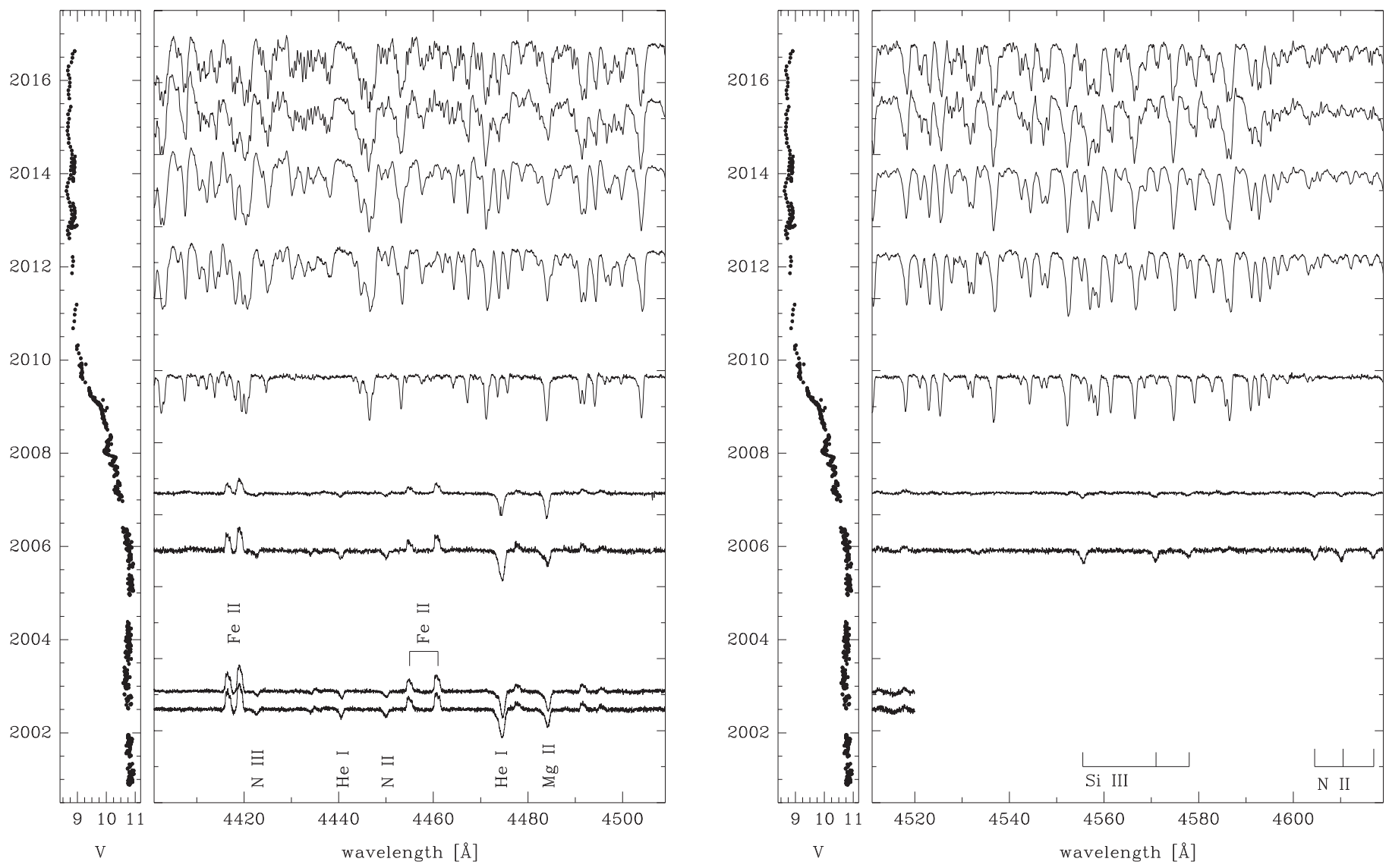

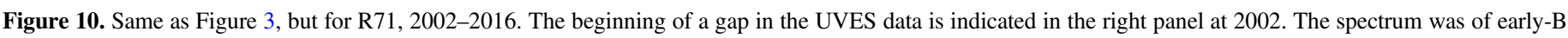

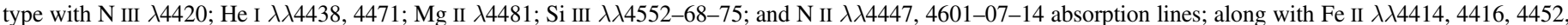

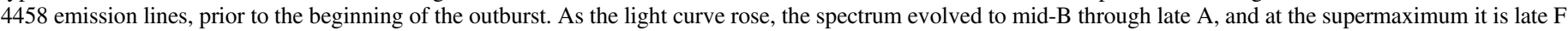
to early $\mathrm{G}$ (see also Figure 9 and text). The major ordinate ticks are separated by 1.0 continuum unit.

and 1950, peaking in about 1914 and 1939, with the second peak being somewhat narrower and higher. Unfortunately, there follows an almost complete data gap until 1970, the latter coinciding with its discovery as an LBV (Thackeray 1974; Wolf 1975). This gap prevents a clear connection between the earlier and later activity, as does another gap between the early 1990s and 2000. Nevertheless, the overall impression is that of a series of outbursts of increasing amplitude from 1900 to the present. Again, is a crisis in progress? It is not unreasonable to project a giant eruption or even a CCSN ensuing; again, see Pastorello et al. (2010).

The recent/current light curve is magnified in Figure 7 . The impressive increase from $V \sim 10.8$ in 2006 (already elevated from minima at 11.2 in Figure 6 ) has leveled off at $\sim 8.8$ by 2012, an unprecedented value in the historical record of R71 (Gamen et al. 2009) where it currently remains. It is worth noting that this peak is identical to the "supermaximum" of R127 between 1988 and 1991 (Walborn et al. 2008 and Section 4.1 below). See also Figure 1 of Szczygieł et al. (2010).

Our complete violet through green low-resolution spectroscopic coverage of R71 from 2009 through 2017 is listed in Table 4 and displayed in Figure 8. A monotonous, late-type pure absorption spectrum is seen that is consistent with the flat supermaximum in $V$ throughout these years (Figure 7). Most of the lines are due to Fe II and other singly ionized metals. Spectral classification is performed from the highresolution data presented next. Surprisingly, the 2017 observation does not show the inverse P Cyg profiles that appeared at longer wavelengths in the high-resolution data discussed below.

Wolf et al. (1981) presented the first high-resolution spectrograms that well characterized the variation from a peculiar A type with P Cyg profiles at the 1973 light maximum to an equally peculiar early-B type in 1981 approaching a minimum. It would be extremely interesting to know whether or not the spectrum became even hotter during the minima later in the 1980s, but we are not aware of any pertinent observations.

Our high-resolution spectroscopic observations of R71 displayed in the following Figures 9-13 are listed in Table 5. As anticipated by the low-resolution data, the blue through green spectra as of 2009 consist almost entirely of pure absorption lines of singly ionized metals, predominantly Fe II and Ti II. The apparent spectral type becomes progressively later during this time period. In Figure 9, we display series of the $4900 \AA$ region containing $\mathrm{Ba}$ II and $\mathrm{Fe}$ II lines, the ratio of which is a sensitive temperature criterion, in both R71 and a supergiant spectral-type sequence from the ESO/UVES/POP resource (Bagnulo et al. 2003) to support an approximate classification of the R71 spectra. The increasing ratio of $\mathrm{Ba}$ II $\lambda 4934$ to Fe II $\lambda 4924$ provides a striking criterion along this sequence. In 2005 and earlier, 

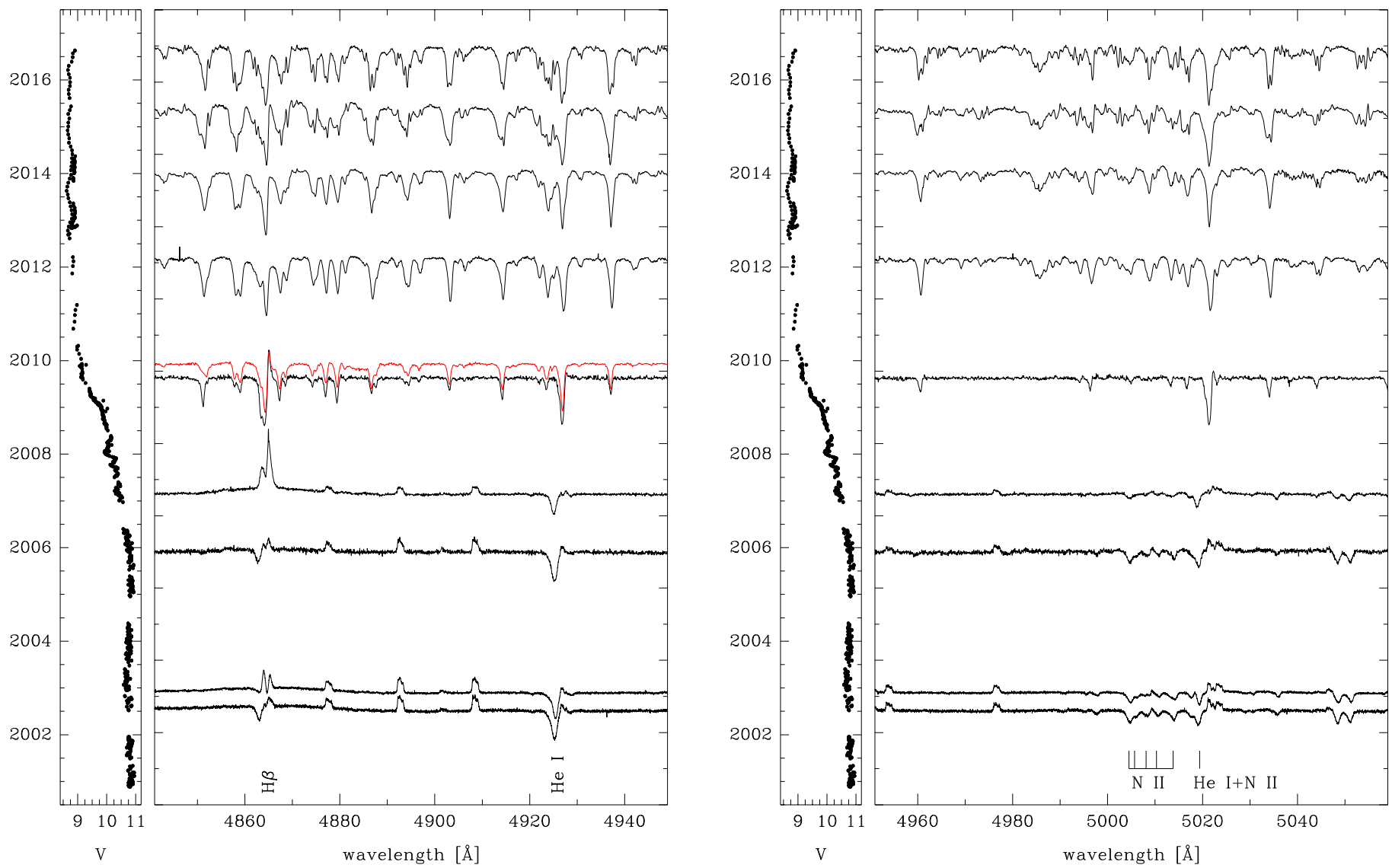

Figure 11. Same as Figure 3, but for the 4800-5000 $\AA$ regions in R71, 2002-2016. The extreme variability of the H $\beta$ profile is remarkable; a second late 2009 observation is included in red for clarity because it changed even between those two. He I $\lambda 4922$ is identified at the left, while the N II $+\mathrm{He}$ I complex at $5000-20 \AA$ at the right is also noteworthy.

R71 had a spectral type earlier than B8, with the only absorption line in this region being He I $\lambda$ 4922. (The 1995 observation is of special interest because it falls in one of the aforementioned photometric coverage gaps; Figure 6.) In 2009, approaching but not quite at its light maximum, R71 had a late-A spectrum. From the unit value of the ratio in the 2012 spectrum, we classify it as F9-G1 at maximum, which is an unprecedentedly late value for R71 or perhaps any LBV outburst observed with this data quality. Of course, this spectral type is merely "equivalent" and does not specify quantitative physical parameters because it does not arise in a stellar photosphere, but from a transient structure of unknown nature in detail. Rather, it serves as a line-content description for comparison with other epochs in R71 and with other LBVs. The spectral type of $\eta$ Carinae became even later during its nineteenthcentury eruption, as recently revealed by its light echoes (Rest et al. 2012). In 2015 and 2016, the absorption lines in R71 become broader and then doubled, indicating some dynamical event in the extremely extended envelope.

Temporal sequences of further interesting features in the spectra of R71 at high resolution are displayed in Figures 10-13. Some salient features are discussed here, while further details are noted in the figure captions.

Figure 12 presents interesting features at yellow and red wavelengths. First, the 5800-5900 $\AA$ region nicely dramatizes the huge contrast between the early 21 st-century minimum spectrum and that of the current superoutburst. The disappearance of the He I line and the appearance of stellar $\mathrm{Na}$ I features are striking. Note also the large changes in the $\mathrm{Ba}$ II profile among the last four observations, from narrow through broad to double or with central emission, to predominantly inverse $\mathrm{P}$ Cyg, diagnosing dynamical events with very small or null changes in the light, as does the extreme variability of the $\mathrm{H} \alpha$ profile. It shows an impressive systematic evolution from predominantly emission line before the outburst, through a P Cyg profile with multiple/ variable absorption components in 2009 during the late rise in light, to a symmetrical albeit variable "Be" absorption with emission wings, or alternatively a broad, centrally reversed emission at maximum. However, as discussed and referenced by Walborn et al. (2015), such a profile does not necessarily correspond to a disk, as it can be produced by radiativetransfer effects in a spherical envelope. As usual, this line will provide vital diagnostics of the unknown LBV outer structure as a function of time, even within the visual light maximum.

In both panels of Figure 12, we have added surprising new data obtained in 2017 January during the final stages of the manuscript, revealing yet another striking behavior of R71: many of the metal lines have developed apparent inverse P Cyg profiles, perhaps smoothly from a central emission component previously interpreted in terms of a double absorption profile. If this phenomenon corresponds to an incipient contraction of the superoutburst envelope, then its velocity at this epoch is $\sim 30 \mathrm{~km} \mathrm{~s}^{-1}$. The emission peaks of 

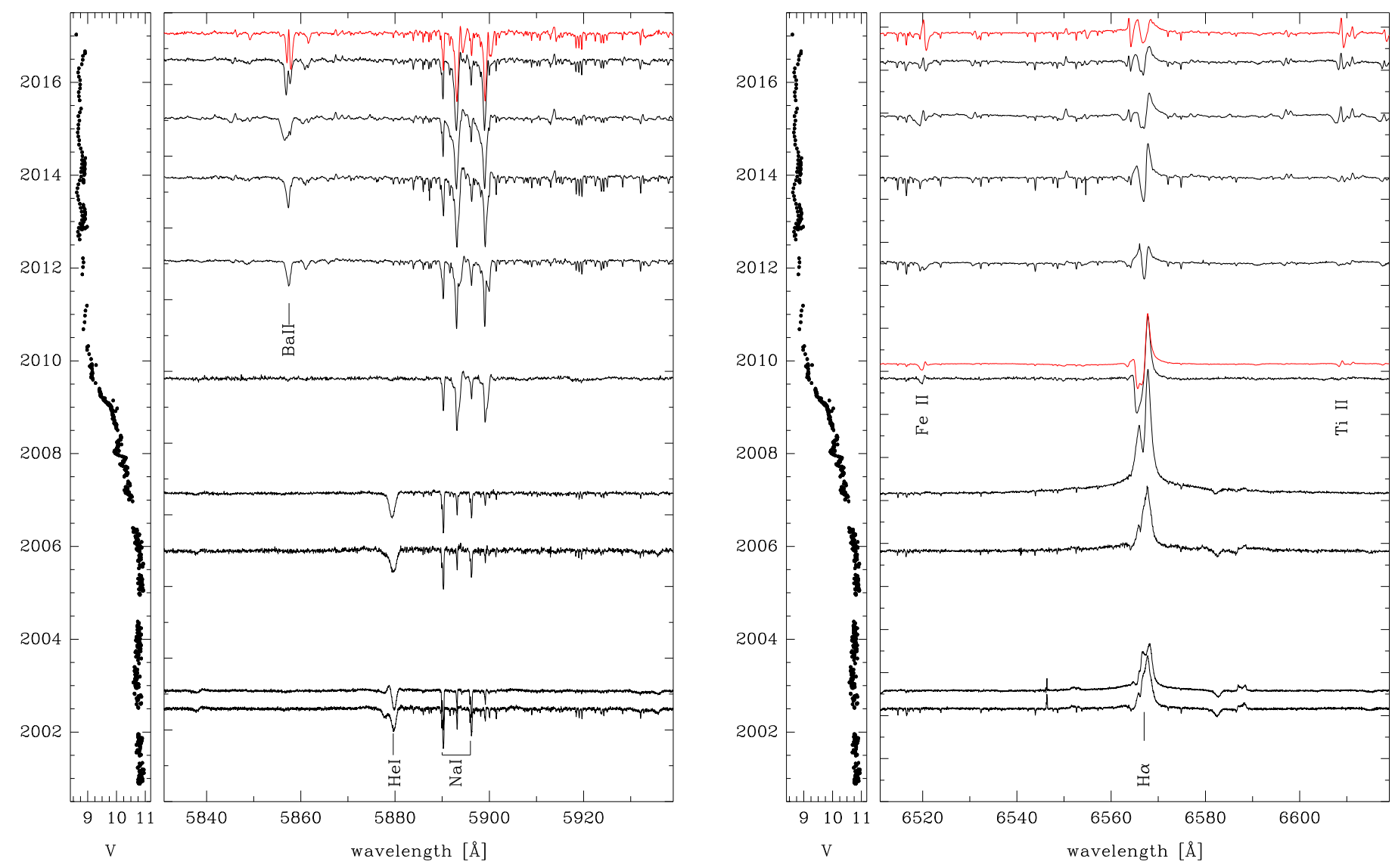

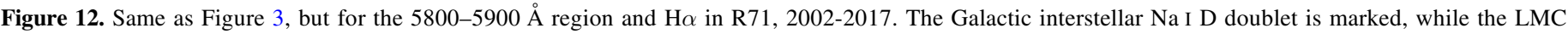

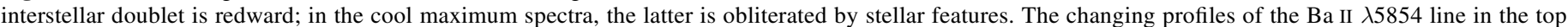

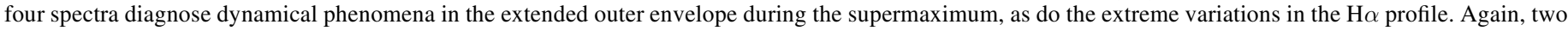

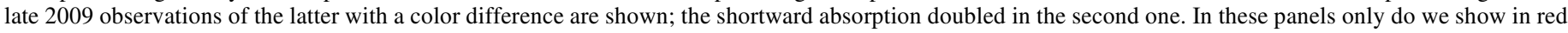

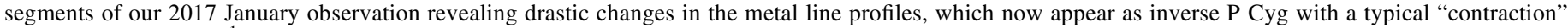

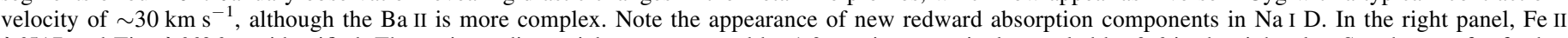

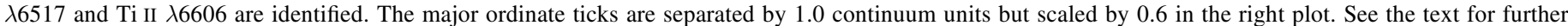
discussion.

these lines have velocities of $\sim 140 \mathrm{~km} \mathrm{~s}^{-1}$, as compared to $\sim 195 \mathrm{~km} \mathrm{~s}^{-1}$ frequently reported for R71, primarily if not entirely from lines of [Fe II] (Feast et al. 1960; Thackeray 1974; Wolf et al. 1981; Mehner et al. 2013). Further investigation is required to determine whether these are profile effects or real velocity differences, whether spatial or temporal, and, in the latter case, whether from binary motion, LBV oscillations, pulsations, or all of the above, which is far beyond the scope of this paper and may well require more intensive coverage than currently available to disentangle. The classification criteria shown in Figure 9 did not change in 2017. The contemporaneous photometric observation also plotted in Figure 12 yields $V \sim 8.6$, which is brighter than the previous value but not inconsistent with the "pulsation" pointed out in the Figure 7 caption. Clearly, continued monitoring is essential. Evidently, identical phenomena during a maximum of S Doradus were reported by Wolf \& Stahl (1990), who also suggested an origin in pulsations.

Finally, Figure 13 shows the surprising appearance and strengthening of the [Ca II] $\lambda \lambda 7291,7324$ emission lines, as discovered and announced by Gamen et al. (2012b) and Walborn et al. (2012) and analyzed by Mehner et al. (2013).
Yet again, comparison with $\eta$ Carinae is called for because Prieto et al. (2014) recently discovered the emergence of these lines during its nineteenth-century outburst, as revealed by its light echoes. These multiple similarities between the behaviors of R71 and $\eta$ Carinae present the prospect that they may well illuminate each other. Might the current giant outburst of R71 be portending an eruption? These lines have also attracted considerable recent interest because of their presence in some luminous red transients (Humphreys et al. 2011) and CCSN or imposters (e.g., Dessart et al. 2009; Smith et al. 2010; Mauerhan et al. 2013b). Presumably, they correspond to line-formation conditions rather than an abundance effect in R71. These lines remained invariant during the drastic changes of 2017 January in the photospheric metal lines, likely indicating that they are formed at a larger radius in the envelope that has not yet been affected. This figure also displays the evolution of the $\lambda \lambda 8500-8600$ Ca II Triplet profiles during this period with P Cyg profiles in 2009. Here, the Ca II lines are well distinguished from the Paschen absorption lines about $3 \AA$ redward in each case in the late-type spectra. 

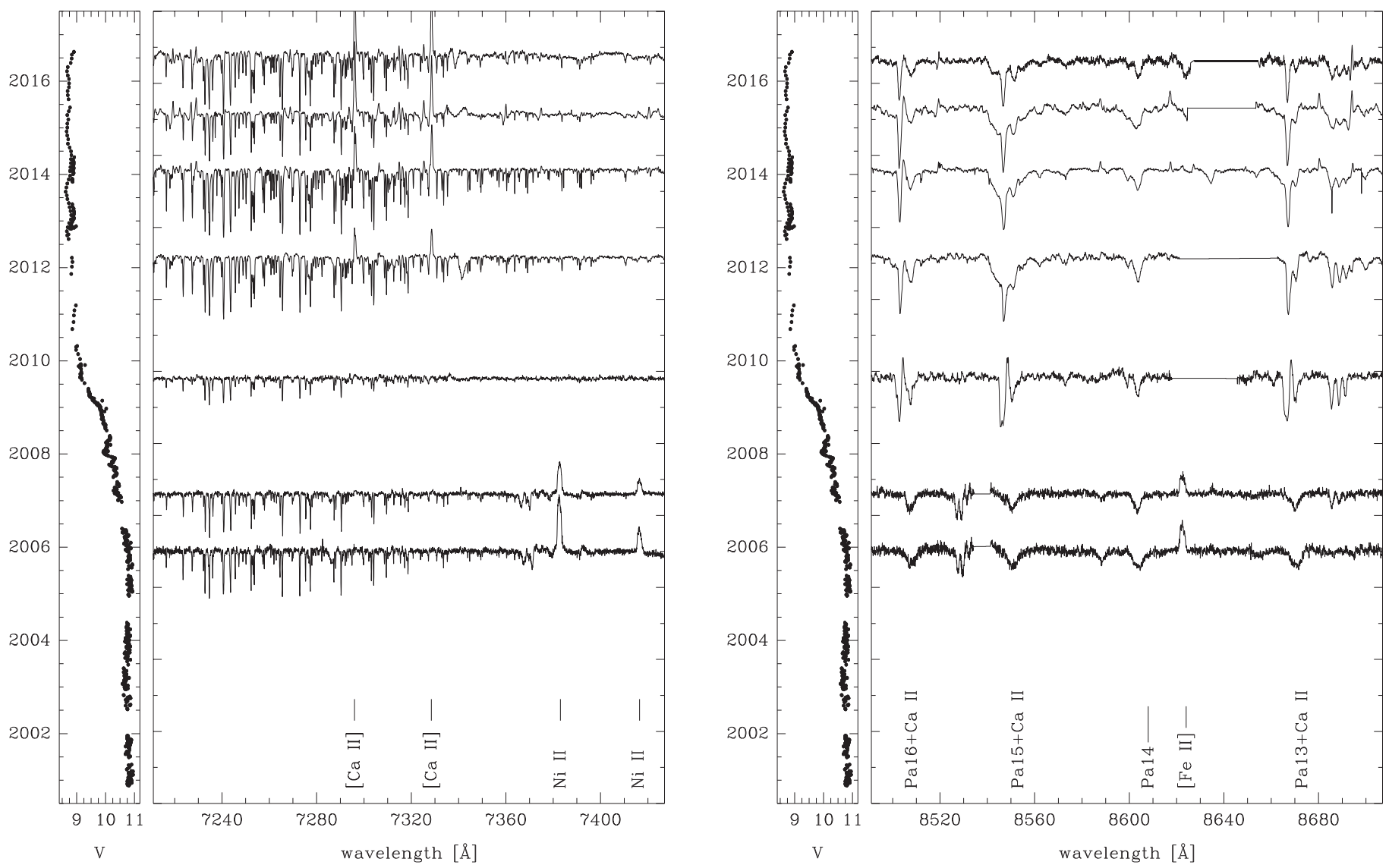

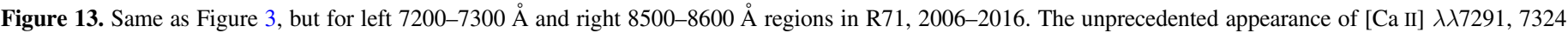

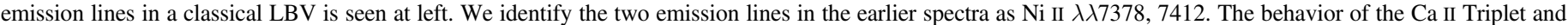

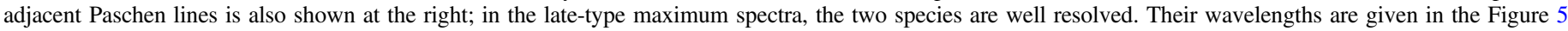
caption.

\subsection{HDE 269582}

The light curve of this less known LMC LBV shows that it is also currently in outburst, although not (yet) as extreme as R127 and R71 (Figure 14). Fortunately, its minimum state is well characterized by both spectroscopy and photometry dating from the 1980s. Most of the trends in the ASAS data for this star, particularly after 2004, are systematic and hence real. There was a historical maximum at $V=9.8$ in 2013, followed by a sharp decline of more than a magnitude by 2015 which has since reversed toward a new maximum.

HDE 269582 was first recognized and discussed as an LBV by Stahl (1986), who provides a good summary of previous knowledge; its variable spectrum was also shown by Bohannan \& Walborn (1989). Crowther \& Smith (1997) show that the Ofpe/WN9 spectrum, which they classfify as WN10h, persisted in 1994 December. A detailed comparison with the light variations was not previously available. Here, for the first time, we show that the hot spectrum corresponds to the quiescent, minimum-light LBV state (Figure 14).

Our low-resolution spectroscopic data (Table 6, Figure 15) show the Crowther \& Smith Ofpe/WN9 spectrum in late 1994, presumably still in the deep minimum observed earlier in the decade, followed by a typical LBV early-B type with strong He I P Cyg profiles and well-marked N II lines in early 2003, just at the start of a minor minimum in the light curve (Figure 14). Then, in late 2009, higher on the light curve, the spectral type is late $\mathrm{B}$; compare to the Mg II $\lambda 4481 / \mathrm{He}$ I $\lambda 4471$ primary classification criterion in that range. Those lines are absent in the 2010 October 26 observation but have returned by 2010 November 27 . Unfortunately, a photometric data gap between early 2010 and late 2012 precludes a detailed comparison between the spectrum and light during that interval. On 2012 September 28, the strongest A-type spectrum in our sequence occurs close to the light curve maximum. Thereafter, the spectrum returns to earlier types as the $V$ light declines. Again, the detailed level of correlation between the spectrum and light is remarkable.

Thus, HDE 269582 becomes a "second R127" for which the full light amplitude and corresponding spectral variation from Ofpe/WN9 to A have been directly observed. However, we cannot exclude the possibility that the outburst will resume toward even brighter $V$ in the near future; note the several smaller precursor maxima prior to the peak of R127 (Wolf et al. 1988; Walborn et al. 2008). As has already been pointed out, a recovery from the 2014-2015 minimum of HDE 269582 is currently underway (Figure 14).

The available high-resolution data (Table 7, Figures 16-18) are of course in agreement with the low-resolution trends, but naturally reveal further details of the varying line profiles at those epochs. The doubling of the N II and Si III absorption lines in the B-type spectrum of 2010 November is noteworthy. 


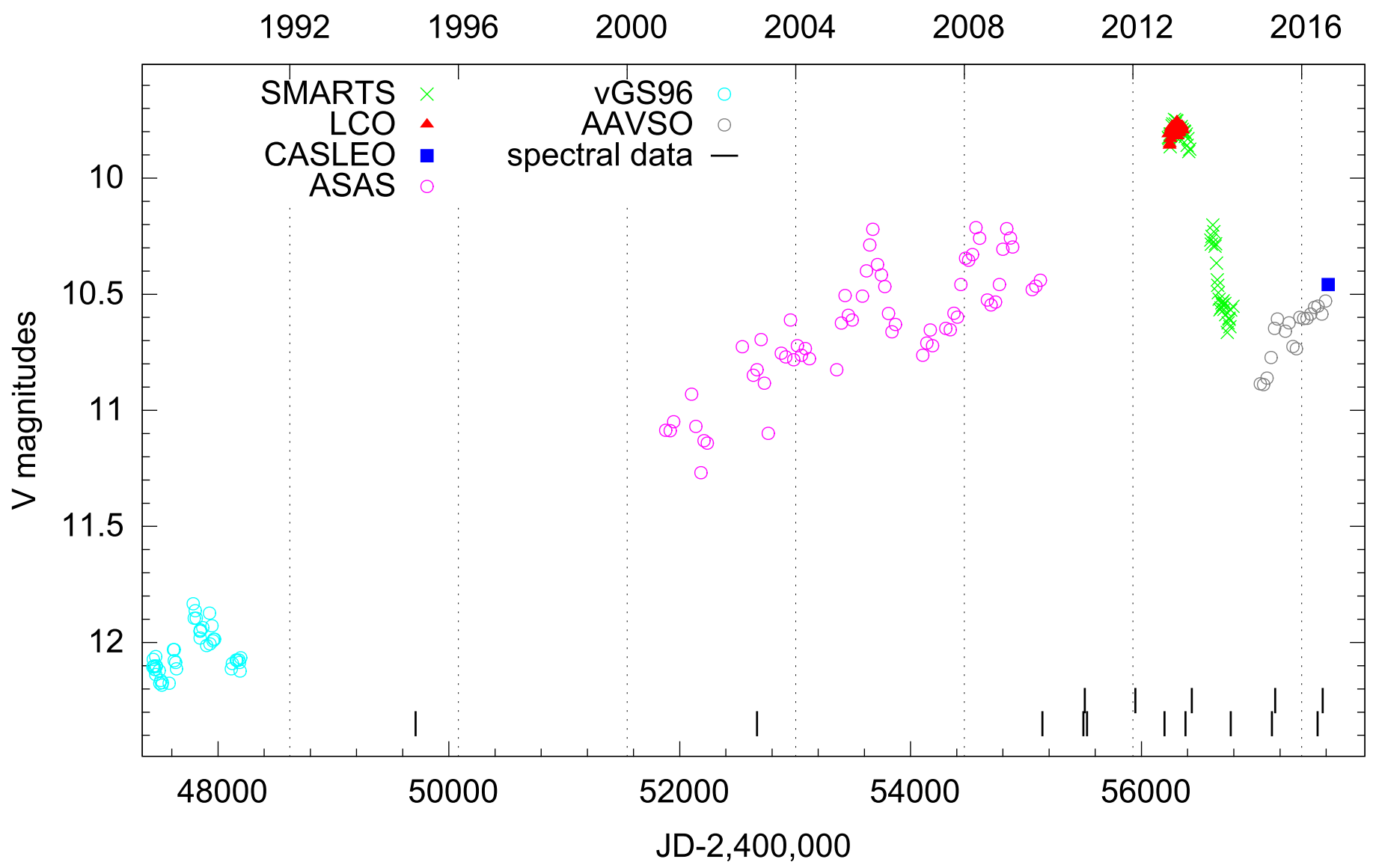

Figure 14. Recent/current visual light curve of HDE 269582. A full range from $V \sim 12$ to 9.8 is newly recognized. vGS96: van Genderen \& Sterken (1996).

Fortunately, the extreme A-type spectra with strong Fe II P Cyg profiles of 2012-2013, which are characteristic of LBV maxima, are well covered, as is the return to B type in 2015-2016. It is all too obvious that monitoring of LBVs must be pursued with the highest possible frequency to adequately know their behavior.

\subsection{HDE 269216}

Ardeberg et al. (1972, running number 173, p. 286) cataloged $V=10.76, B-V=+0.08$, and a spectral type of B7: Iae with the remark, "This P Cygni spectrum seems variable. Hydrogen emission (down to $\mathrm{H} \epsilon$ ) was strong in Feb. 1969 but much weaker in 1970 November. Emission of He I 4026-4471 suspected." The only detailed discussion of this interesting but little-known object is provided by Prinja \& Schild (1991), who first suggested that it may be an LBV. It is located in the small association Lucke-Hodge 39, which likewise interestingly contains one of the original Ofpe/WN9 stars, HDE 269227 (Walborn 1977, 1982; Schild 1987). Essentially identical luminosities and masses $\left(\sim 25 M_{\odot}\right)$ were derived for the two stars which, as pointed out, are surprisingly low. HDE 269216 came to our attention through I. D. Howarth's unpublished survey of LMC OB spectra, in which it clearly shows LBV Balmer profiles.

The light curve as shown in Figure 19 shows definite variability, with an overall rise from $V$ of $10.5-11$ in the early 1990s to a historical maximum of 9.8 in 2016. In agreement with the light curve, here we document the first observed LBV outburst of HDE 269216 by means of our two lowresolution observations in 2014 and 2016, shown in Figure 20; we also show the 2006 observation by Howarth that he has kindly made available (Table 8). The appearance of the first two spectra is consistent with previous descriptions, i.e., a late-B supergiant with peculiar P Cyg Balmer and $\mathrm{Fe}$ II profiles, although the stronger $\mathrm{He} \mathrm{I}$ lines in 2006 show a somewhat earlier type than in 2014. The systematic decline of the narrow emission at $\mathrm{H} \gamma$ among the three epochs is noteworthy. $\mathrm{H} \beta$ shows the the broad wings discussed by Walborn et al. (2015) with an asymmetry ascribed to a diffuse interstellar band in the longward wing; unusually, strong and broad Balmer emission extends down to $\mathrm{H} \delta$, as in the Galactic LBV HR Carinae (Hutsemékers \& Van Drom 1991). In contrast, the 2016 spectrum has metamorphosed into an AF type dominated by a plethora of strong Fe II absorption lines, although the broad Balmer emission remains.

In Figures 21-22, we display segments of our sole highresolution observation in 2016 July (included in Table 8), which so far as we know is also the only such observation in existence to date. Of course, the spectral content is similar to that of the June low-resolution observation where they overlap. Fe II and Ti II absorption lines are double in this spectrum. 


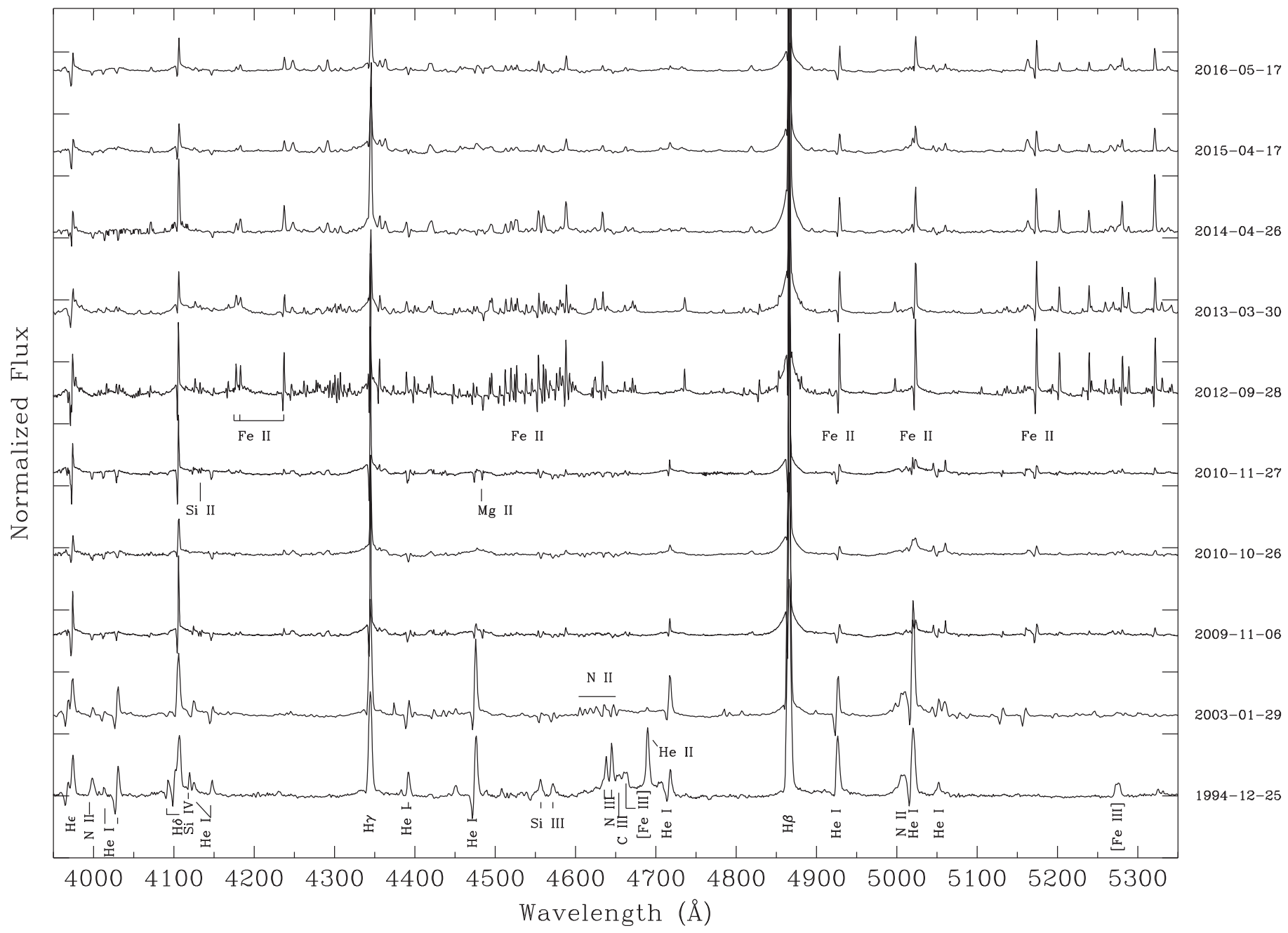

Figure 15. Low-resolution violet-green spectroscopy of HDE 269582, 1994-2016. A remarkable evolution from Ofpe/WN9 through early then late B to A and back is documented in tight correlation with the light curve (Figure 14). The ordinate ticks are separated by 1.30 continuum units.

\section{5. $R 143$}

R143 is located in an evolved association immediately SE of the main 30 Doradus Nebula (Walborn \& Blades 1997). Feast et al. (1960) reported an F-supergiant spectrum, but Parker (1993) observed it to be 1.4 magnitudes fainter with a late-B spectrum (see also Walborn 1997), establishing its LBV nature. Smith et al. (1998; see also Weis 2003) subsequently found ejected nitrogen-rich circumstellar nebulosity, indicative of a larger, earlier eruption.

Parker (1993) quotes $V=10.62, B-V=+0.74$ from Westerlund (1961). The precise date of the observation is not known, but presumably it is approximately contemporaneous with the spectroscopy of Feast et al. (1960). In contrast, Parker measured $V=12.01, B-V=+0.44$ from data obtained in late 1985 . The photometry we have been able to obtain is assembled into a largely undersampled light curve in Figure 23. The level of the ASAS data is uncertain by at least a few tenths of a magnitude because the large aperture contains numerous other stars, at least one of which is variable, so large corrections of about $+0.8 \mathrm{mag}$ have been applied and extreme, random outliers temporally near very different values have been discarded. Nevertheless, there appears to have been a gradual brightening of about $0.5 \mathrm{mag}$ between 1982 and 2016. However, there have evidently also been significant excursions around that trend, as implied by variations in our sparse spectroscopy which we discuss next.

Our three low-resolution spectroscopic observations from 2012-2014 are listed in Table 9 and displayed in Figure 24. They show significant changes: from a peculiar A type with marked Fe II absorption and P Cygni profiles in the first observation to late-B or somewhat earlier A types subsequently. Unfortunately, we have not located any photometry during these years. Note that the low-resolution observation from January 1993 shown by Walborn (1997) has a definite B9 Ip type.

Our high-resolution data from 1998 through 2008, and then in 2016, are listed in Table 10 and displayed in Figures 25-26. Ironically, they are completely disjoint from the low-resolution data and show He I lines that do not appear to be entirely a resolution effect, indicating a late-B type. The implication is that the star was hotter and presumably fainter during the highresolution observations, at least before the low-resolution ones. The 2008 observation has the weakest He I lines, which may be consistent with a possible shallow maximum in the ASAS 
Table 4

Low-resolution Spectroscopic Observations of R71

\begin{tabular}{lcll}
\hline \hline Observation Date & HJD-2450000.0 & Instrument & PI/Observer \\
\hline 2009 Aug 29 & 5072.8 & IMACS & N. Morrell \\
2009 Nov 06 & 5141.8 & MagE & P. Massey/N. Morrell \\
2010 Nov 27 & 5527.8 & MagE & P. Massey/N. Morrell \\
2013 Mar 30 & 6381.5 & B\&C & N. Morrell \\
2014 Apr 27 & 6774.4 & B\&C & N. Morrell \\
2015 Apr 15 & 7127.5 & B\&C & N. Morrell \\
2016 May 21 & 7530.4 & B\&C & R. Barbá \\
2017 Feb 07 & 7791.5 & MagE & P. Massey/N. Morrell \\
\hline
\end{tabular}

Table 5

High-resolution Spectroscopic Observations of R71

\begin{tabular}{|c|c|c|c|}
\hline $\begin{array}{l}\text { Observation } \\
\text { Date }\end{array}$ & $\begin{array}{c}\text { HJD- } \\
2400000.0\end{array}$ & Instrument & PI/Observer \\
\hline 1995 Oct 03 & 49994.0 & UCLES $^{\mathrm{a}}$ & S. Chapman \\
\hline $2002 \mathrm{Jul} 23$ & 52478.9 & UVES $^{\mathrm{b}}$ & T. Szeifert \\
\hline 2002 Aug 03 & 52489.9 & UVES & T. Szeifert \\
\hline 2002 Aug 12 & 52498.9 & UVES & T. Szeifert \\
\hline 2002 Sep 04 & 52521.9 & UVES & T. Szeifert \\
\hline 2002 Sep 10 & 52527.9 & UVES & T. Szeifert \\
\hline 2002 Sep 18 & 52535.8 & UVES & T. Szeifert \\
\hline 2002 Sep 22 & 52539.8 & UVES & T. Szeifert \\
\hline 2002 Sep 30 & 52547.9 & UVES & T. Szeifert \\
\hline 2002 Oct 06 & 52553.9 & UVES & T. Szeifert \\
\hline 2005 Dec 12 & 53716.2 & FEROS & $\begin{array}{l}\text { M. Kraus/M. Bor- } \\
\text { ges Fernandes }\end{array}$ \\
\hline 2007 Feb 22 & 54153.2 & FEROS & B. Davies \\
\hline 2009 Aug 26 & 55069.9 & $\begin{array}{r}\text { du Pont/ } \\
\text { echelle }\end{array}$ & N. Morrell \\
\hline 2010 Jan 08 & 55204.6 & MIKE & $\begin{array}{l}\text { M. Phillips/N. } \\
\text { Morrell }\end{array}$ \\
\hline 2011 Feb 13 & 55605.6 & FEROS & R. Barbá \\
\hline 2012 Feb 19 & 55976.5 & $\begin{array}{r}\text { du Pont/ } \\
\text { echelle }\end{array}$ & N. Morrell \\
\hline 2014 Jan 18 & 56675.9 & MIKE & R. Barbá \\
\hline 2015 May 12 & 57155.5 & $\begin{array}{r}\text { du Pont/ } \\
\text { echelle }\end{array}$ & N. Morrell \\
\hline 2016 Jun 30 & 57569.9 & $\begin{array}{r}\text { du Pont/ } \\
\text { echelle }\end{array}$ & N. Morrell \\
\hline 2017 Jan 13 & 57766.6 & $\begin{array}{r}\text { du Pont/ } \\
\text { echelle }\end{array}$ & $\begin{array}{l}\text { N. Morrell/G. } \\
\text { Ferrero }\end{array}$ \\
\hline
\end{tabular}

Notes.

${ }^{a}$ University College London Echelle Spectrograph at the Anglo-Australian Telescope.

${ }^{\mathrm{b}}$ Ultraviolet and Visual Echelle Spectrograph at the ESO Very Large Telescope. Mehner et al. (2013).

photometry. So there should have been an at least comparable maximum during the photometrically missing 2012-2014 interval. However, it is noteworthy that all of the highresolution data show an apparent inverse $\mathrm{P}$ Cygni profile in the sensitive He I $\lambda 5876$ line, indicating no drastic change in the LBV envelope structure during those years, which is in agreement with the relatively invariant $\mathrm{H} \alpha$ profiles. On the other hand, the He I $\lambda 4922$ profiles are quite different, complex, and variable, which is reminiscent of the large differences among He I profiles in Oe spectra (Sota et al. 2011, 2014; Gamen et al. 2012a).
Table 6

Low-resolution Spectroscopic Observations of HDE 269582

\begin{tabular}{lcll}
\hline \hline Observation Date & HJD-2400000.0 & Instrument & PI/Observer \\
\hline 1994 Dec 25 & 49711.0 & RGO $^{\mathrm{a}}$ & P. Crowther \\
2003 Jan 29 & 52668.6 & WFCCD $^{\text {b }}$ & N. Morrell \\
2009 Nov 06 & 55141.8 & MagE & P. Massey/N. Morrell \\
2010 Oct 26 & 55495.7 & B\&C & N. Morrell \\
2010 Nov 27 & 55527.8 & MagE & P. Massey/N. Morrell \\
2012 Sep 28 & 56198.9 & MagE & P. Massey/N. Morrell \\
2013 Mar 30 & 56381.5 & B\&C & N. Morrell \\
2014 Apr 26 & 56773.5 & B\&C & N. Morrell \\
2015 Apr 17 & 57129.5 & B\&C & N. Morrell \\
2016 May 17 & 57530.4 & B\&C & N. Morrell
\end{tabular}

Notes.

${ }^{a}$ Royal Greenwich Observatory Spectrograph at the Anglo-Australian Telescope.

b $2.5 \mathrm{~m}$ du Pont Telescope at LCO.

Table 7

High-resolution Spectroscopic Observations of HDE 269582

\begin{tabular}{lcll}
\hline \hline Observation Date & HJD-2400000.0 & Instrument & PI/Observer \\
\hline 2010 Nov 10 & 55510.7 & du Pont/echelle & N. Morrell \\
2012 Jan 21 & 55947.5 & MIKE & N. Morrell \\
2013 May 24 & 56437.4 & du Pont/echelle & N. Morrell \\
2015 May 15 & 57158.4 & du Pont/echelle & N. Morrell \\
2016 Jul 01 & 57570.9 & du Pont/echelle & N. Morrell \\
\hline
\end{tabular}

\section{Discussion}

\subsection{Phase Identity}

In the preceding descriptions of the varying LBV spectra in comparison with their light curves, the detailed reproducibility of the correlations has been emphasized. Of course, that property of LBV variations has long been known, although perhaps not at the level of detail for even relatively minor variations as we have demonstrated. Here we extend the discussion to the close relationships in that regard even among different objects, which may have significant implications for their largely unknown physics. The correspondences between approximate spectral types and the $V_{0}$ magnitudes are summarized in Table 11, including a few historical references prior to the majority of the entries found in this paper. The specific criteria for these spectral classifications are then listed in Table 12 because all of the spectra are peculiar and the standard criteria are frequently compromised by superimposed or blended emission lines of the same or other species; again, as emphasized in the detailed classification of R71 above, these types do not have the same physical significance as those of normal stellar atmospheres, but rather are useful for describing and intercomparing the content and ionization of the variable spectra of an individual LBV or different ones. The reddenings or extinctions have been taken from the literature as noted in Table 1; when only the former was given, $R=3$ was necessarily adopted. The strong relationships between these types and the $V_{0}$ magnitudes indicate that all of the objects have similar intrinsic magnitudes and that the 

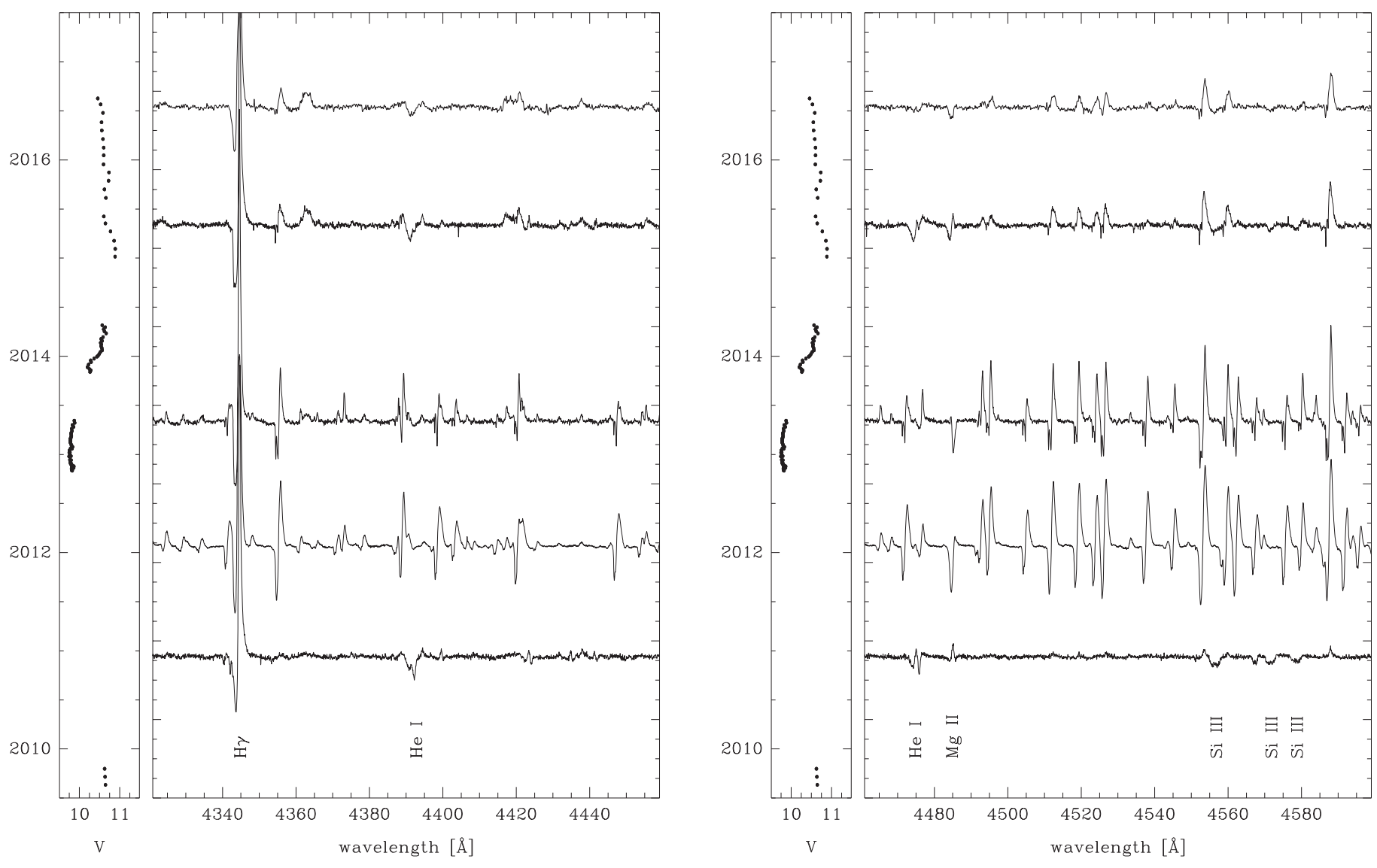

Figure 16. Same as Figure 3, but for the (left) $4400 \AA$ and (right) $4500 \AA$ regions in HDE 269582, 2010-2016. He I $\lambda \lambda 4387,4471$ and Mg II $\lambda 4481$ are identified in the 2010 B-type state. Note the double Si III $\lambda \lambda 4552-68-75$ absorption lines in 2010; the separations are $1.4 \AA$ or $90 \pm 10 \mathrm{~km} \mathrm{~s}{ }^{-1}$. See Walborn et al. (2008) and references therein for the wavelengths of the Fe II lines in the A-type states. The ordinate major ticks are separated by 1.0 continuum units.

Table 8

Spectroscopic Observations of HDE 269216

\begin{tabular}{lccc}
\hline \hline Observation Date & HJD-2450000.0 & Instrument & PI/Observer \\
\hline 2006 Nov 29/Dec 07 & 4073.0 & $\mathrm{AA} \Omega^{\mathrm{b}}$ & I. Howarth \\
2014 Apr 26 & 6773.5 & $\mathrm{~B} \& \mathrm{C}$ & N. Morrell \\
2016 May 21 & 7530.5 & $\mathrm{~B} \& \mathrm{C}$ & N. Morrell \\
2016 Jul 24c & 7593.8 & $\mathrm{du}$ Pont/echelle & N. Morrell
\end{tabular}

Notes.

${ }^{\mathrm{a}}$ This observation is a combination of three individual spectrograms obtained on 2006 Novmeber 29 and December 3 and 7; Julian Dates have been averaged.

${ }^{\mathrm{b}}$ Howarth \& Walborn (2012).

c High res.

correlations are independent of any mass differences among them.

First, we note that the $V_{0}$ magnitudes of the two observed Ofpe/WN9 quiescent states, for R127 and HDE 269582, are essentially identical, as are those of the three A or F-type "supermaxima" for R127, R71, and S Doradus (with the last being from Massey 2000). Moreover, the intermediate spectra and magnitudes are also tightly correlated, as shown in
Table 9

Low-resolution Spectroscopic Observations of R143

\begin{tabular}{lcll}
\hline \hline Observation Date & HJD-2450000.0 & Instrument & PI/Observer \\
\hline 2012 Sep 28 & 6198.9 & MagE & P. Massey/N. Morrell \\
2013 Mar 30 & 6381.5 & B\&C & N. Morrell \\
2014 Apr 26 & 6773.5 & B\&C & N. Morrell
\end{tabular}

Table 10

High-resolution Spectroscopic Observations of R143

\begin{tabular}{lclc}
\hline \hline Observation date & HJD-2400000.0 & Instrument & PI/Observer \\
\hline 1998 Nov 29 & 51146.6 & FEROS & $\ldots$ \\
2003 Dec 04 & 52977.6 & FEROS & 2P2 Team 22 \\
2007 Feb 22 & 54153.6 & FEROS & B. Davies \\
2008 Jul 23 & 54670.9 & du Pont/echelle & R. Barbá \\
2016 Jul 24 & 57593.9 & du Pont/echelle & N. Morrell \\
\hline
\end{tabular}

Figure 27. Nevertheless, an important difference to understand is why some of these states are dominated by emission or P Cyg profiles of the metal lines while others (for the same and different objects) have pure absorption-line spectra. Line doubling in some spectra is another phenomenon requiring 

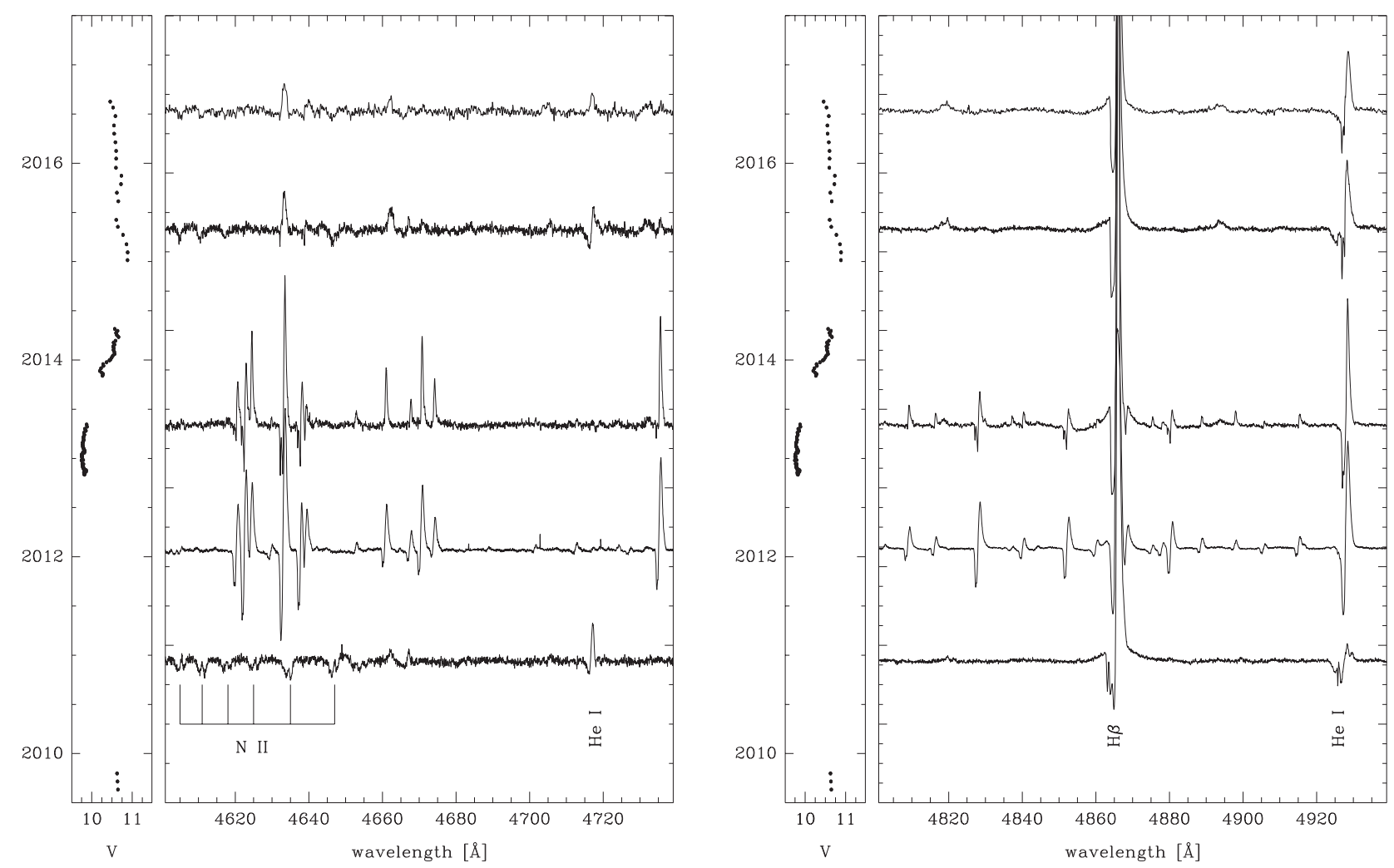

Figure 17. Same as Figure 3, but for the (left) 4600-4700 $\AA$ and (right) 4800-4900 $\AA$ regions in HDE 269582, 2010-2016. Note the double N II $\lambda \lambda 4601-07-14-21-31-43$ absorption lines in 2010 with separations of $1.5 \AA$ or $\sim 100 \mathrm{~km} \mathrm{~s}^{-1}$, similar to Si III in the previous figure. He I $\lambda 4713$ is also identified. The strongest Fe II line at left in the 2012-13 maximum state is $\lambda 4629$. At right, the He I $\lambda 4922$ feature of 2010 is replaced by Fe II $\lambda 4924$ in 2012-13, while the 2015-16 feature is likely a blend of the two. The ordinate major ticks are separated by 1.0 continuum units in both panels, but the vertical plot scale is compressed by a factor of 2 in the right panel.
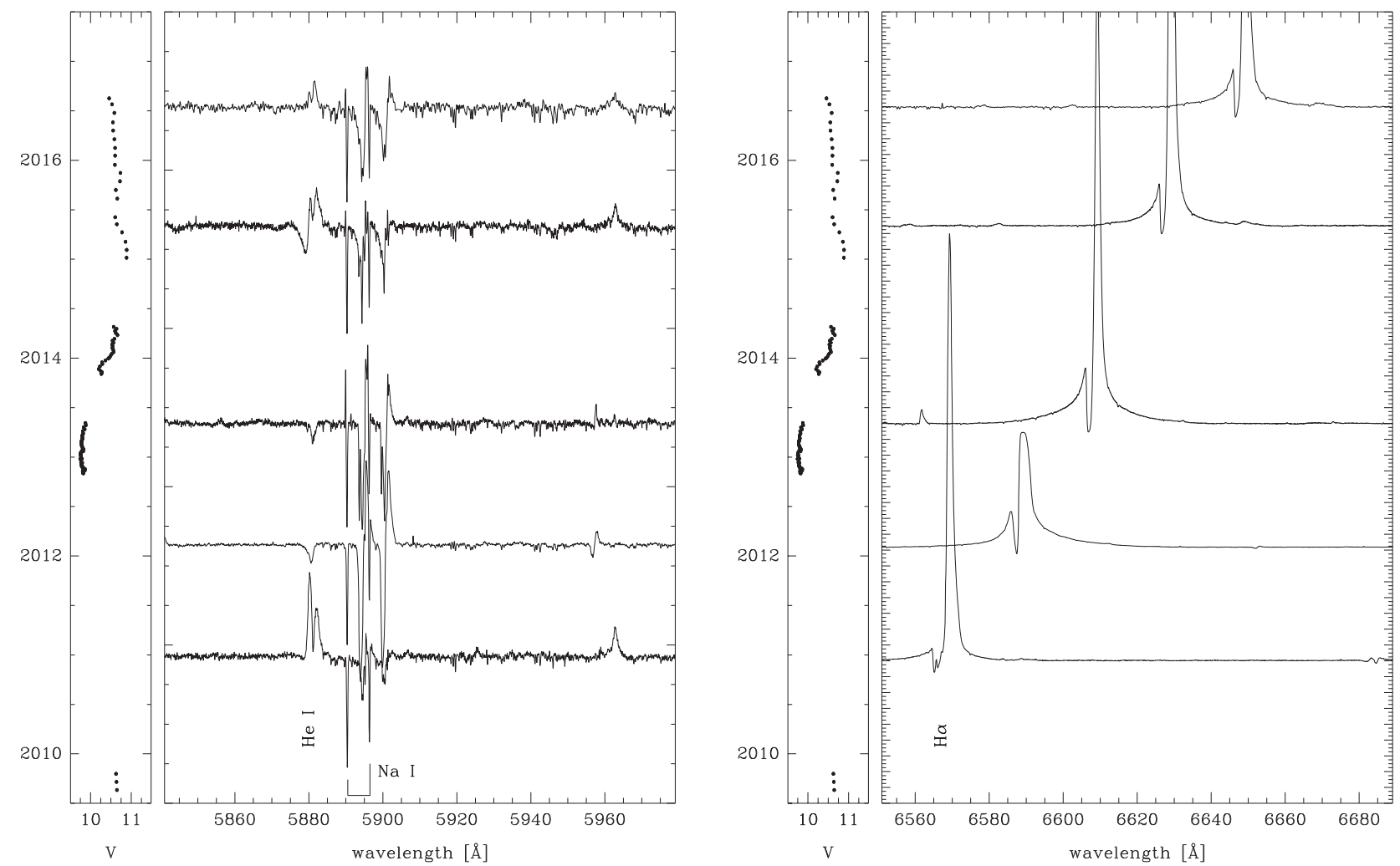

Figure 18. Same as Figure 3, but for (left) the 5800-5900 $\AA$ and (right) $6500 \AA$ Aregions in HDE 269582, 2010-2016. The variations of the sensitive He I $\lambda 5876$ line are remarkable. Complex, variable stellar and circumstellar Na I D features are present at all epochs which obscure the LMC interstellar components; the Galactic interstellar doublet is marked. The $\mathrm{H} \alpha$ profiles have been arbitrarily shifted horizontally to avoid superpositions; the wavelength scale in the right panel applies only to the lowest spectrogram. The ordinate major ticks are separated by 1.0 continuum units in both panels, but the vertical plot scale is compressed by a factor of 10 in the right panel. The $\mathrm{H} \alpha$ emission peaks in 2013, 2015, and 2016 are at 31.2, 46.3, and 37.5 continuum units relative to zero, respectively. 
The Astronomical Journal, 154:15 (26pp), 2017 July

Walborn et al.

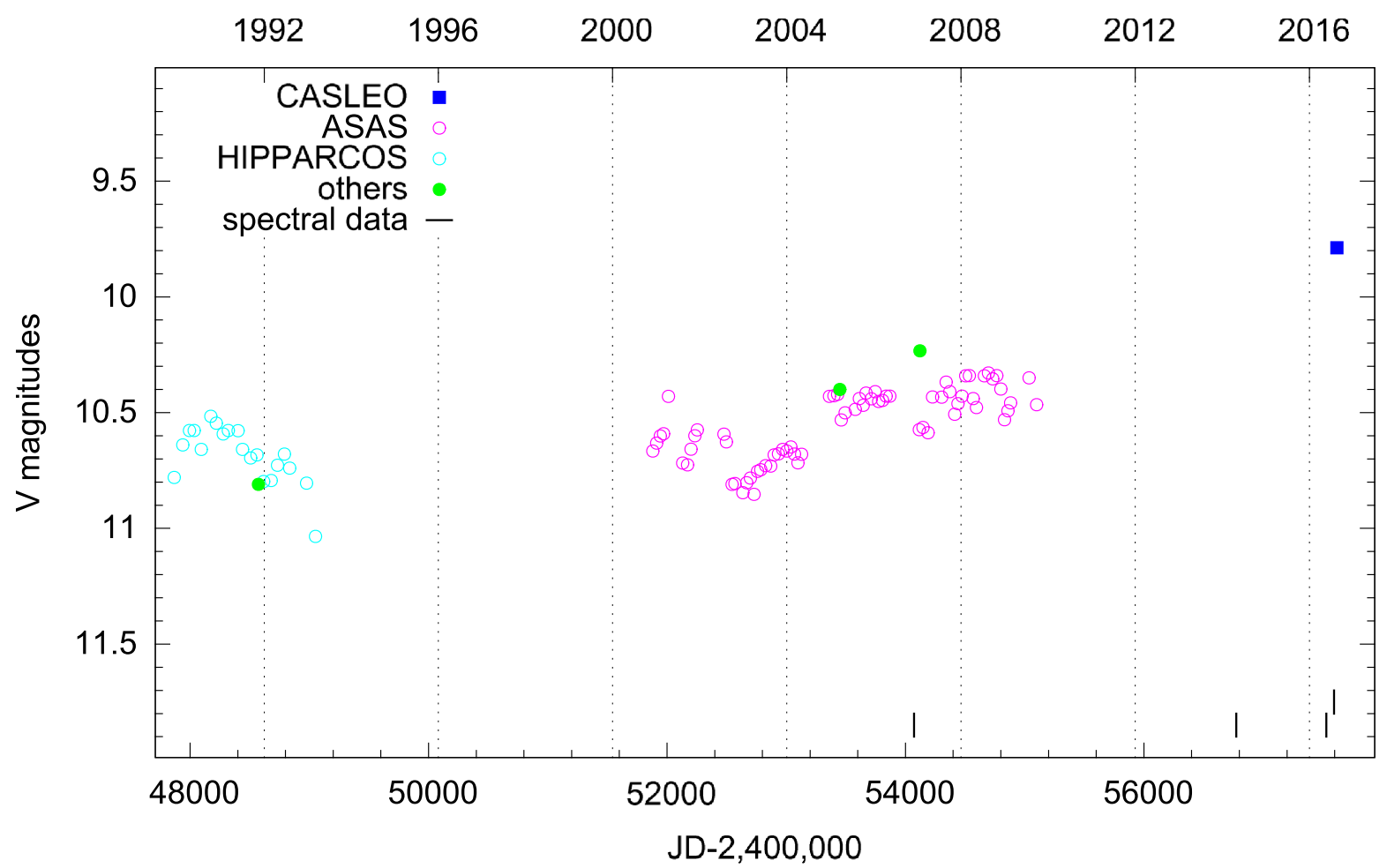

Figure 19. Light curve of HDE 269216, 1990-2016. Hipparcos: van Leeuwen et al. (1998). Other sources: 1991, Gummersbach et al. (1995) 2005, Munari et al. (2009) 2007, Butters et al. (2010).

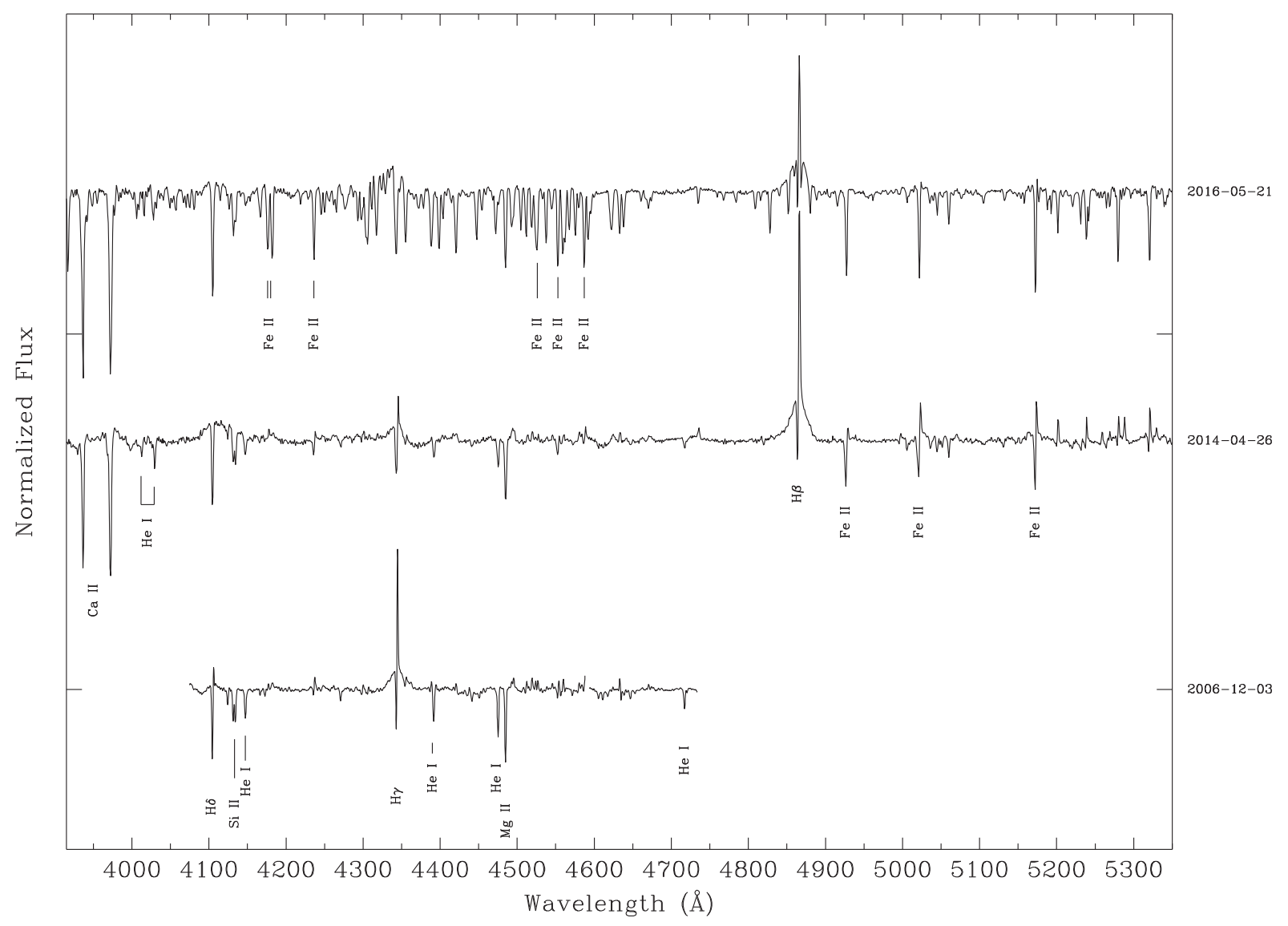

Figure 20. Low-resolution violet-green spectroscopy of HDE 269216 in 2006, 2014, and 2016. The extreme transformation between the latter two epochs represents the first spectroscopically observed LBV outburst of this object. The narrow $\mathrm{H} \gamma$ emission-line intensity is inversely correlated with the spectral types (see text). The ordinate ticks are separated by 0.70 continuum units. 

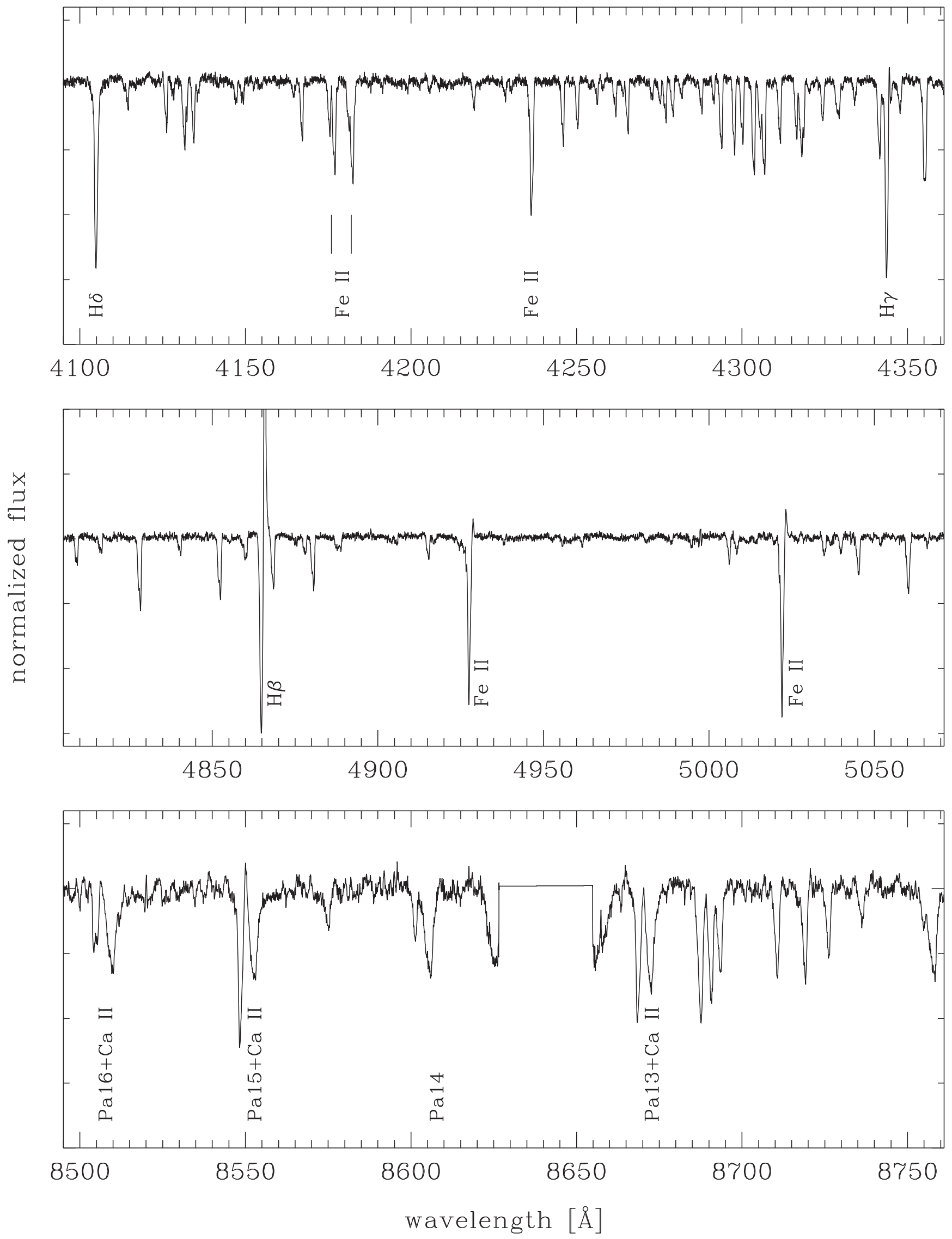

Figure 21. Spectral segments in our sole high-resolution observation of HDE 269216 in 2016 July corresponding to the A outburst type. The identified Fe II lines are $\lambda \lambda 4173-79,4233,4924,5018$. The wavelengths of the Paschen and Ca II Triplet lines are in the Figure 5 caption. The minor ordinate ticks are separated by 0.25 continuum units. 

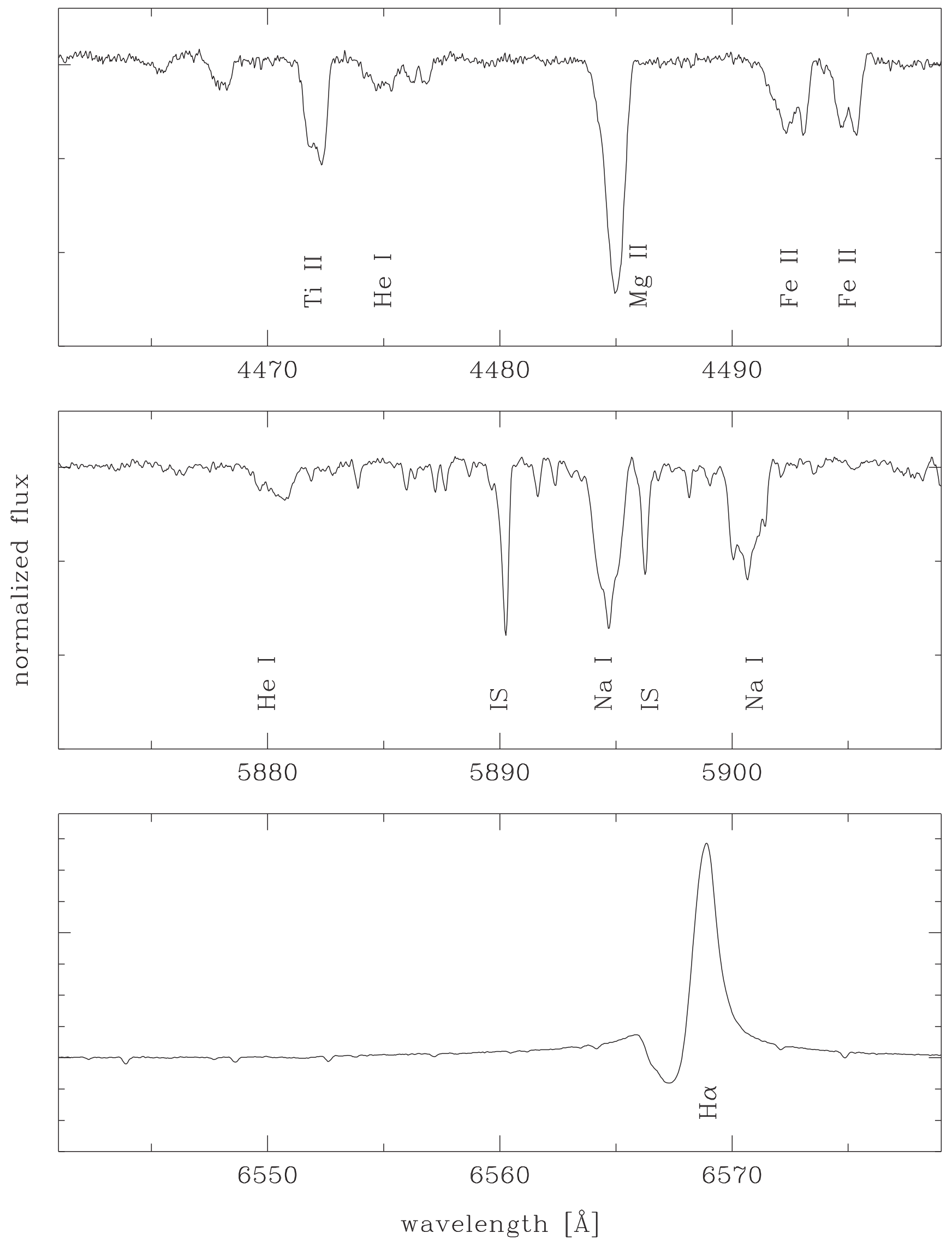

Figure 22. Spectral segments in our sole high-resolution observation of HDE 269216 in 2016 July corresponding to the A outburst type, although very weak He I $\lambda \lambda 4471,5876$ features remain visible. Fe II and Ti II lines are doubled by $0.6 \AA$ or $45 \pm 6 \mathrm{~km} \mathrm{~s}^{-1}$. Galactic interstellar Na I D components are marked by "IS," while the corresponding redshifted LMC features are blends of stellar and interstellar contributions. The minor ordinate ticks are separated by 0.25 continuum units, which are more compressed for $\mathrm{H} \alpha$ to show the full profile, corresponding to scaling by 0.4 . 


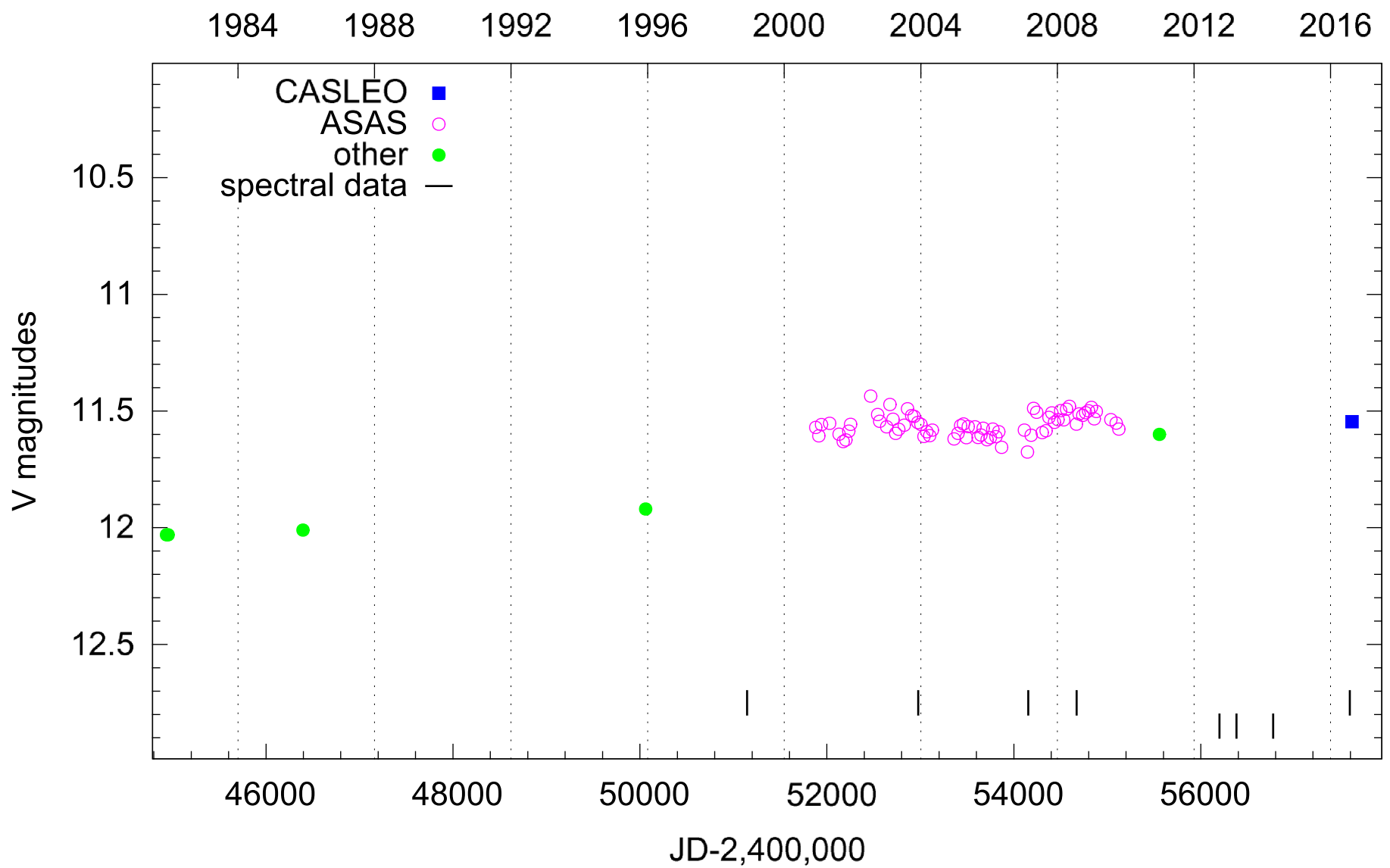

Figure 23. Light curve of R143, 1981-2016. Other sources: 1981, Clayton \& Martin (1985) 1985, Parker (1993) 1995, Smith et al. (1998) 2010, C. Evans (2016, private communication).

explanation. In any event, we have established that the typical full range of LBV outbursts is 3 magnitudes, as opposed to the smaller values that are sometimes quoted.

All of these objects except for R71 are in OB clusters or associations (Table 1). In LBV HR diagrams, R71 is usually shown as less luminous and massive than the others (e.g., Walborn et al. 2015). Moreover, the outbursts are shown as horizontal displacements at constant bolometric luminosity. However, the current superoutburst of R71 raises serious questions about its parameters and behavior: either it has increased in luminosity, or the previous values were underestimated (see also Mehner et al. 2013). The latter explanation could be related to an underestimate of its (gray?) extinction by the circumstellar dust producing its unique IR excess (Bonanos et al. 2009). Might the dust have been dissipated by the current outburst? A detailed study of its past and current IR behavior will be of interest.

As noted in its presentation above, the mass derived for HDE 269216 is suprisingly low, yet its (few) entries in Table 11 are similar to those for the other objects. Pending further observations of this little-studied object, and in particular whether it will proceed to a brighter maximum (and similarly for HDE 269582), one may speculate whether LBV outbursts produce a funneling effect such that objects of different masses reach similar observational parameters. Related questions are as follow: the significance of the numerous oscillations and inversions of smaller amplitudes that LBVs display; the likelihood that their extreme states may not be achieved (and thus observed) for decades or centuries; and the possibility that they may become "stuck" in particular states with relatively small excursions for very extended periods. Examples of the last phenomenon would be $\mathrm{S}$ Doradus near its maximum since discovery, $\mathrm{P}$ Cygni in an intermediate early-B state since its seventeeth-century eruption, and perhaps even $\eta$ Carinae which was reported at second to fourth apparent magnitude (far too bright for a normal star in its association) for centuries prior to its nineteenth-century giant eruption. Clearly, we have much yet to learn observationally before hoping to understand LBVs theoretically. Despite these complications, however, the reproducibility of the standard outburst behavior, both for a given LBV and among all of them, as has also been emphasized in this section, provides hope that there is a well defined mechanism susceptible to eventual elucidation.

\subsection{Present and Future Highlights}

This paper has presented a detailed morphological overview of our extensive LMC LBV spectroscopic and photometric data sets, which has provided several surprises. They include the following: multiple continuing oscillations of R127 subsequent to its recent superoutburst; major longterm variations of R71 during the first half of the twentieth 


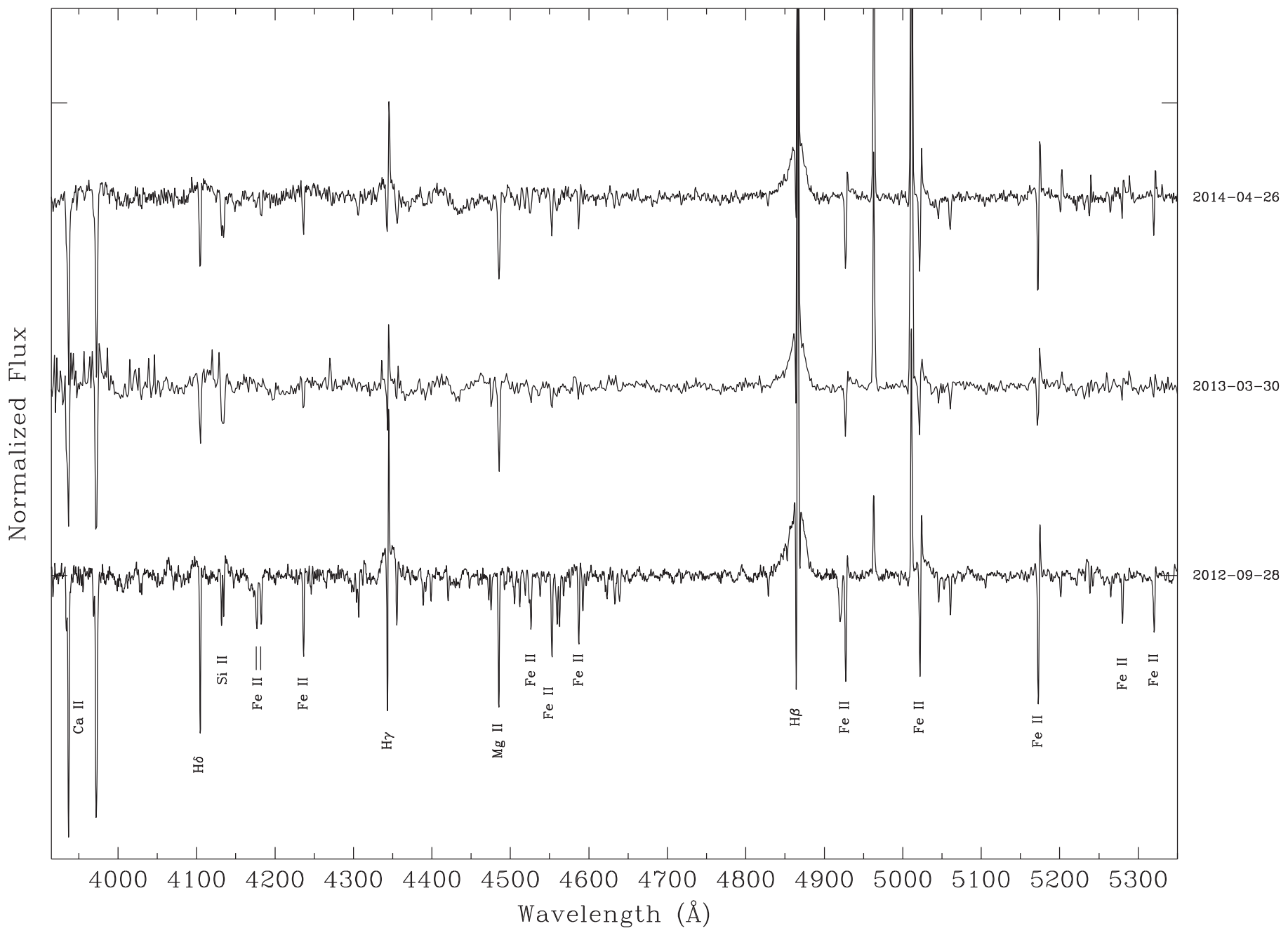

Figure 24. Low-resolution violet-green spectroscopy of R143, 2012-2014. There are significant variations from type A to late B and back to earlier A. The H $\gamma$ emission strength appears to correlate with the spectral types, athough there is a significant nebular contribution from 30 Doradus, as seen in the [O III] $\lambda \lambda 4959,5007$ lines. The ordinate ticks are separated by 0.40 continuum units.

century, the appearance of [Ca II] emission lines during its current supermaximum with unprecedentedly late spectral type, and the recent appearance of inverse P Cyg profiles in metal lines; a faint Ofpe/WN9 quiescent state of HDE 269582 followed by an extended, major LBV outburst; and the first spectroscopically observed LBV outburst of HDE 269216. Yet much further analysis of these data beyond the scope of this paper remains to be carried out, including but not limited to radial-velocity measurements to investigate flows and multiple line components, searches for short (days to a few years) periodic variations that are suggested visually in some data, and eventually integrated astrophysical investigations of the phenomena to further advance our understanding of these mysterious and significant late stages of massive stellar evolution.

We are grateful to the late Albert Jones of New Zealand for alerting us to the current outbursts of R71 and HDE 269582 based on his assiduous visual monitoring. Our highresolution spectroscopy includes observations by the OWN Survey (Barbá et al. 2010). We acknowledge Jesús Maíz
Apellániz (Centro de Astrobiología, Madrid), Emilio Alfaro, and Alfredo Sota (both Instituto de Astrofísica de Andalucía) for LCO $2.5 \mathrm{~m} \mathrm{B \& C}$ data acquired in joint runs with the Galactic O-Star Spectroscopic Survey (Maíz Apellániz et al. 2011). We thank Paul Crowther (University of Sheffield) for the minimum state spectroscopic observation of HDE 269582, Ian Howarth (University College London) for our "discovery" spectrogram of HDE 269216 from his extensive unpublished LMC survey, and Roberta Humphreys (University of Minnesota) for a CTIO observation of R127. We also thank Gabriel Ferrero (UNLP) for several spectroscopic and photometric observations at LCO and CASLEO; and Cristina Putkuri for assistance with echelle observations and reductions at LCO. We are grateful to the staffs of LCO, ESO/La Silla, and CASLEO for their professional support, and to the Carnegie Supernova Project for time and photometric observations at LCO. Observing time on the SMARTS Consortium $1.3 \mathrm{~m}$ telescope at CTIO was secured under the Chilean Program CL-12B-0001. We have made use of data from the UVES Paranal Observatory Project (ESO DDT Program ID 

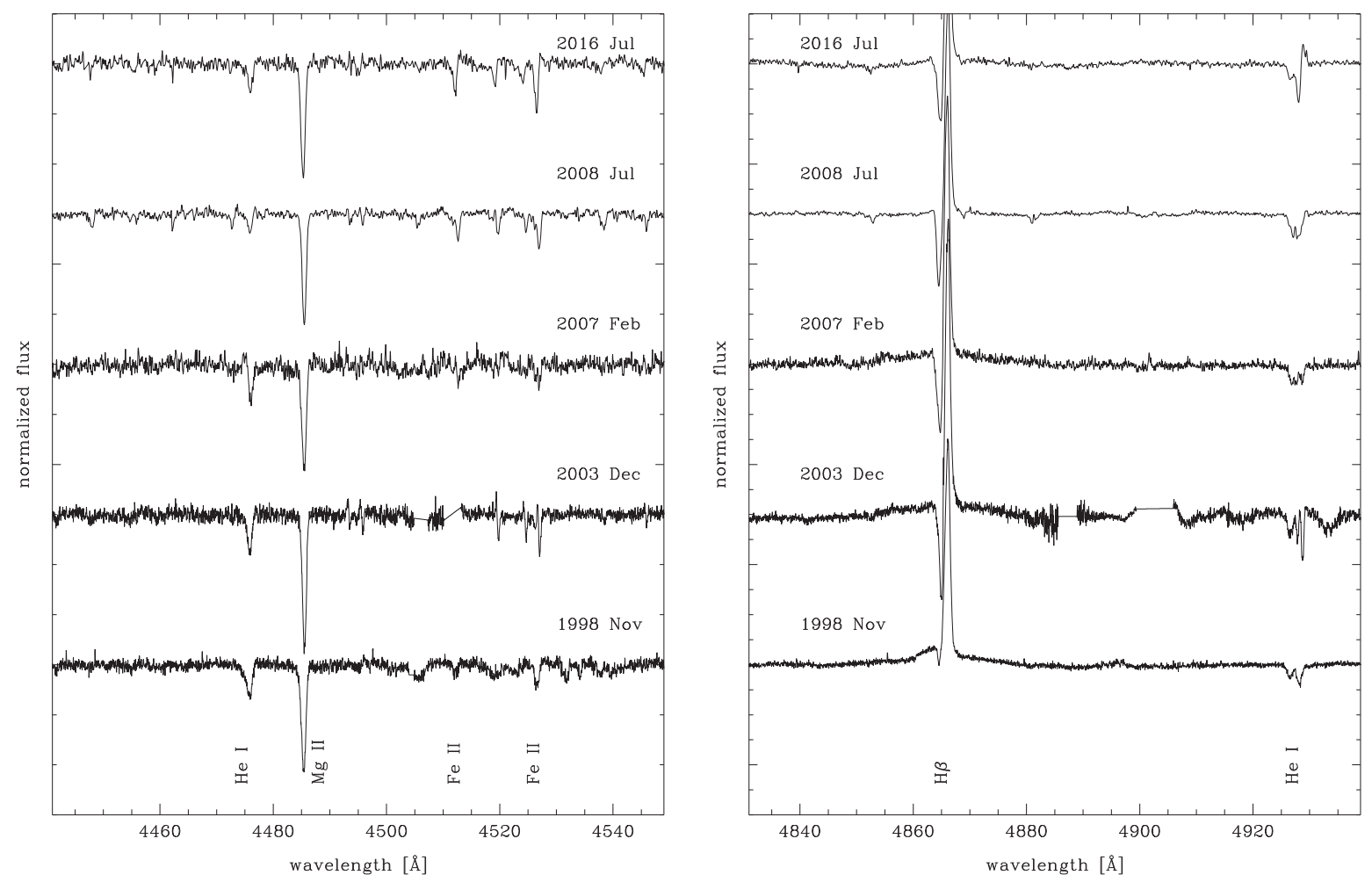

Figure 25. High-resolution spectroscopy of R143 at 4400-4500 $\AA$ (left) and 4800-4900 ̊ (right), 1998-2016. The He I $\lambda 4471$ strength relative to Mg II $\lambda 4481$ indicates a late-B type, except perhaps in 2008, which may be early A. Note the weak inverse P Cyg profile in 2007. The entirely different and highly variable profiles of He I $\lambda 4922$ present a remarkable contrast. The major ordinate ticks are separated by 1.0 continuum units in both panels, albeit more compressed at right.
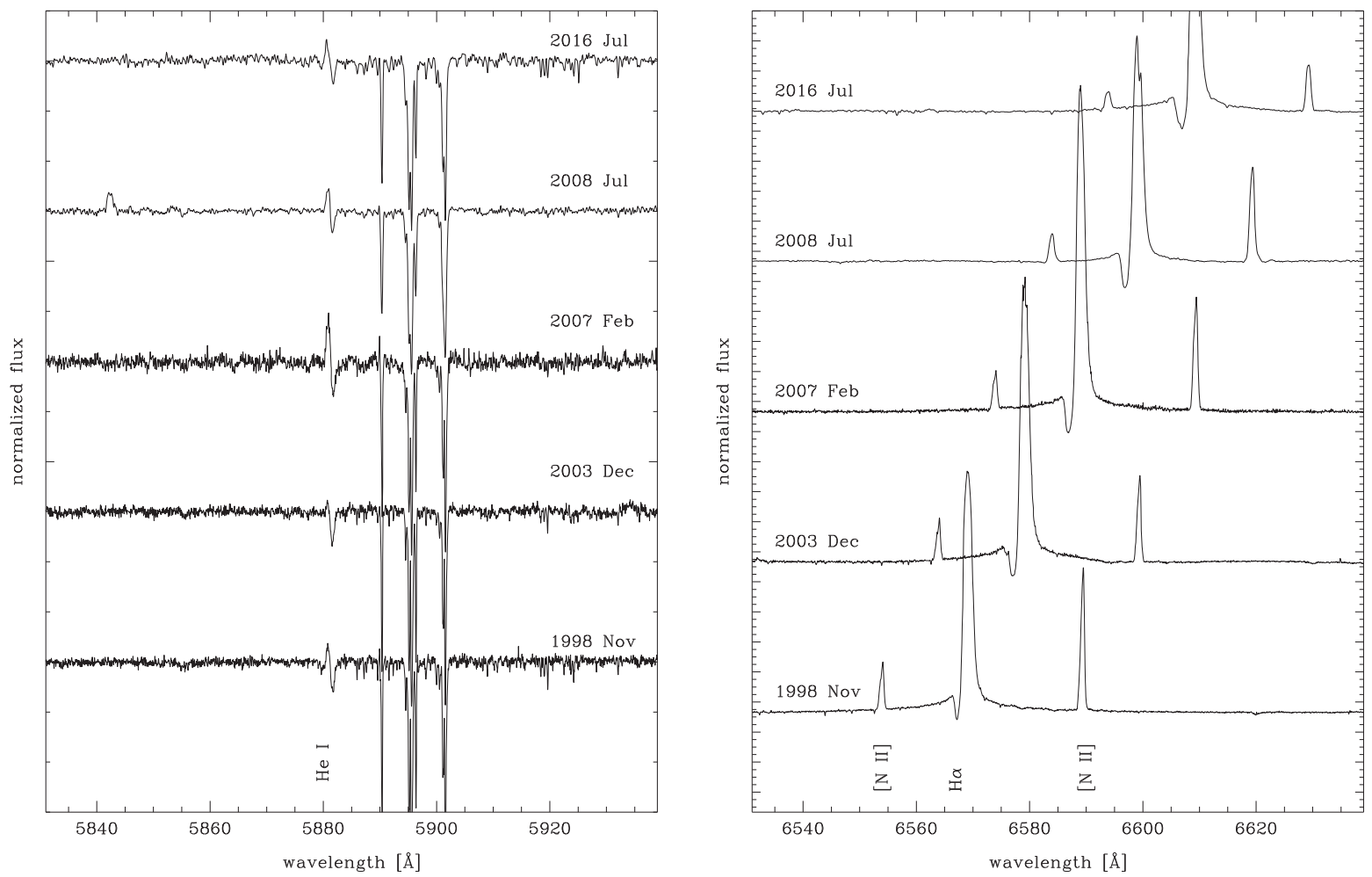

Figure 26. High-resolution spectroscopy of R143 at 5800-5900 A (left) and $6500 \AA$ (right), 1998-2016. He I $\lambda 5876$ has an inverse P Cyg profile throughout, although it is strongest in 2007 (when $\lambda 4471$ in the previous figure also had one). The Galactic interstellar (at 5890 and $5896 \AA$ ) and LMC blended stellar/interstellar (redshifted by $\sim 5 \AA$ ) $\mathrm{Na}$ I D doublets are strong. The $\mathrm{H} \alpha$ profiles have been arbitrarily shifted horizontally to avoid superpositions; the wavelength scale in the right panel applies only to the lowest spectrogram. The $\mathrm{H} \alpha$ and [N II] features are stellar/nebular and circumstellar/nebular blends, respectively. The major ordinate ticks are separated by 1.0 continuum units in both panels, albeit substantially more compressed at the right. The $2016 \mathrm{H} \alpha$ emission peaks at 8.3 continuum units above zero (essentially the same as in 2008). 


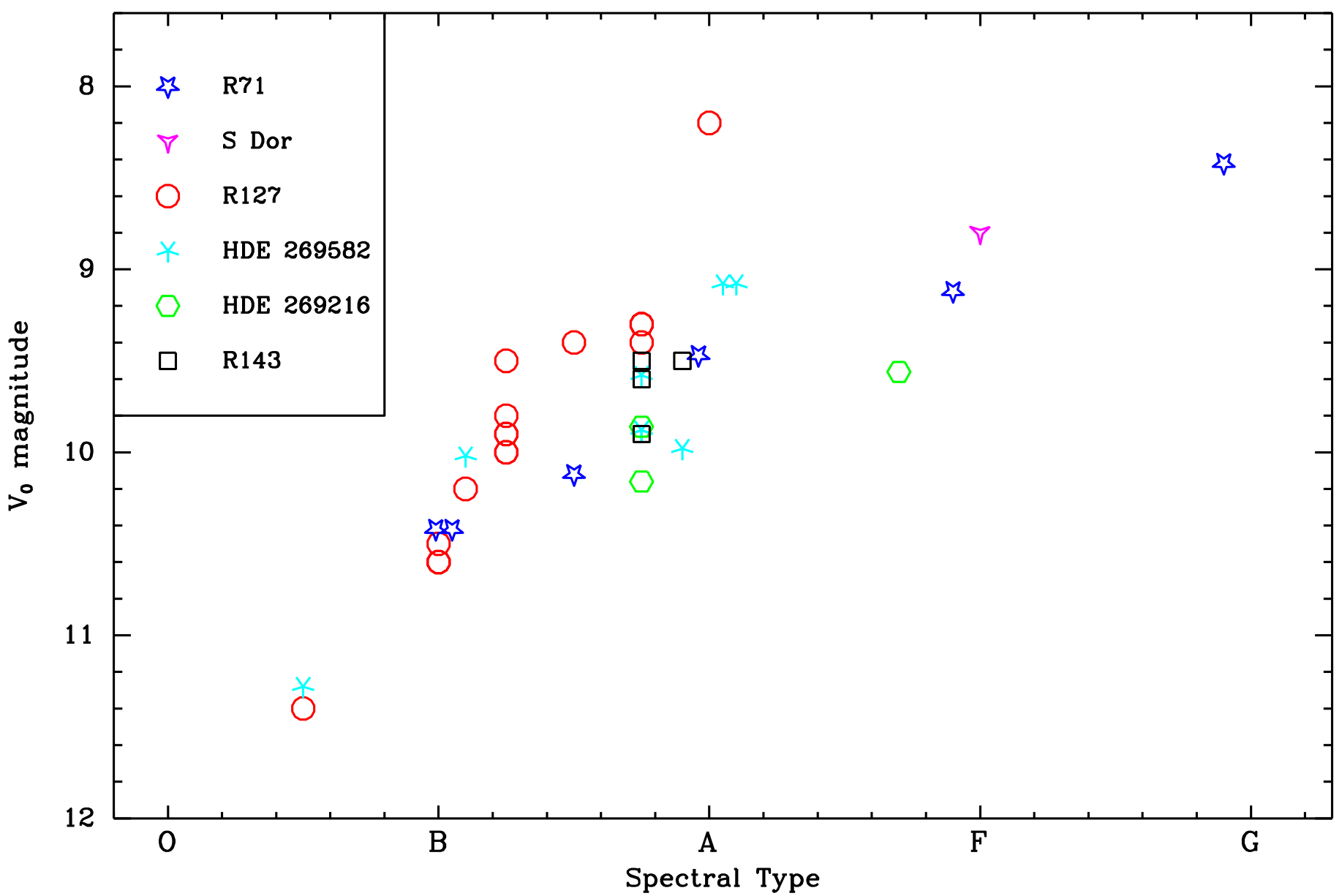

Figure 27. Global $V_{0}$ magnitude vs. spectral-type correlation for LMC LBVs. Note the close agreement of the two Ofpe/WN9 and three supermaximum states observed. Intermediate states between the extremes are also highly correlated.

266.D-5655, Bagnulo et al. 2003). The DASCH project at Harvard University is grateful for partial support from NSF grants AST-0407380, AST-0909073, and AST-1313370. This paper makes use of WASP data (Butters et al. 2010) from the Exoplanet Archive, operated by the California Institute of Technology under contract with NASA. We acknowledge with thanks the variable star observations from the AAVSO International Database contributed by observers worldwide and used in this research. The 2017 January photometric observation of R71 was obtained remotely with the SARA Observatory $0.61 \mathrm{~m}$ telescope at CTIO, which is owned and operated by the Southeastern Association for Research in Astronomy (saraobservatory.org). STScI is operated by AURA, Inc. under NASA contract NAS 5-26555; partial publication support was provided by the STScI Director's Discretionary Research Fund. RHB acknowledges FONDECYT/Chile grant 1140076. RCG and EFL were supported by grant PIP 112-201201-00298 from CONICET/Argentina. An anonymous referee provided some useful comments. We also thank Andrea Mehner for kindly bringing to our attention a flaw in the 2002 UVES data that had led to a spurious result, now removed from this paper.
Table 11

LBV $V$ Magnitudes as a Function of Spectral Type

\begin{tabular}{|c|c|c|c|c|}
\hline Epoch $^{\mathrm{a}}$ & $\mathrm{SpT}^{\mathrm{b}}$ & $V$ & $V_{0}$ & LC Phase \\
\hline \multicolumn{5}{|l|}{ R127 } \\
\hline$\Leftarrow 1978^{\mathrm{c}}$ & Ofpe/WN9 & 12 & 11.4 & quiescent \\
\hline $1988-91^{\mathrm{c}}$ & A em & 8.8 & 8.2 & supermax \\
\hline $2008 \mathrm{Jan}$ & early B & 11.2 & 10.6 & deep min \\
\hline 2008 Apr & early $B$ & 11.2 & 10.6 & deep $\min$ \\
\hline 2009 Apr & late $\mathrm{B}$ ? & 10 & 9.4 & $\max$ \\
\hline $2009 \mathrm{Jul}$ & late $\mathrm{B}$ ? & $9.9 ?$ & 9.3 & $\max$ \\
\hline 2009 Aug & late B & $9.9 ?$ & 9.3 & $\max$ \\
\hline 2009 Nov & mid-B & $10 ?$ & 9.4 & $\max$ \\
\hline 2010 Oct & B & 10.5 & 9.9 & shallow min \\
\hline 2010 Nov & $\mathrm{B}$ & 10.6 & 10.0 & shallow min \\
\hline 2012 Jun$^{*}$ & $\mathrm{~B}$ & 10.1 & 9.5 & $\max$ \\
\hline 2013 Mar & $\mathrm{B}$ & 10.6 & 10.0 & shallow min \\
\hline 2014 Jan* $^{*}$ & $\mathrm{~B}$ & 10.4 & 9.8 & shallow max \\
\hline 2014 Apr & $\mathrm{B}$ & 10.5 & 9.9 & declining \\
\hline 2015 Apr & early B & 11.1 & 10.5 & deep $\min$ \\
\hline 2016 May & early B & 10.8 & 10.2 & intermed min next cycle \\
\hline \multicolumn{5}{|l|}{ R71 } \\
\hline $1973^{\mathrm{c}}$ & $\mathrm{A} 0 \mathrm{e}$ & 9.85 & 9.48 & $\ldots$ \\
\hline $2002^{*}$ & early B & 10.8 & 10.43 & $\ldots$ \\
\hline
\end{tabular}


Table 11

(Continued)

\begin{tabular}{|c|c|c|c|c|}
\hline Epoch $^{\mathrm{a}}$ & $\mathrm{SpT}^{\mathrm{b}}$ & $V$ & $V_{0}$ & LC Phase \\
\hline $2005^{*}$ & early B & 10.8 & 10.43 & $\ldots$ \\
\hline $2007^{*}$ & mid-B & 10.5 & 10.13 & $\cdots$ \\
\hline $2009^{*}$ & late A & 9.5 & 9.13 & $\cdots$ \\
\hline $2012-17$ & F9-G1 abs & 8.8 & 8.43 & supermax \\
\hline \multicolumn{5}{|c|}{ HDE 269582} \\
\hline $1984-94^{\mathrm{c}}$ & Ofpe/WN9 & 12 & 11.28 & quiescent \\
\hline 2003 Jan & early B & 10.8 & 10.08 & rising \\
\hline 2009 Nov & late $\mathrm{B}$ & 10.3 & 9.58 & rising \\
\hline 2010 Oct & B & $?$ & & rising? \\
\hline 2010 Nov & mid-B & $?$ & & rising? \\
\hline 2012 Sep & A em & 9.8 & 9.08 & $\max$ \\
\hline 2013 Mar & A em & 9.8 & 9.08 & $\max$ \\
\hline 2014 Apr & late B & 10.6 & 9.88 & declining near shallow min \\
\hline 2015 Apr & BA & 10.7 & 9.98 & rising from shallow min \\
\hline 2016 May & late B & 10.6 & 9.88 & rising from shallow min \\
\hline \multicolumn{5}{|c|}{ HDE 269216} \\
\hline $2006 \mathrm{Dec}$ & late B & 10.4 & 10.16 & rising \\
\hline 2014 Apr & late B & 10.1: & 9.86 & rising? \\
\hline 2016 May & AF abs & 9.8 & 9.56 & hist max \\
\hline \multicolumn{5}{|l|}{ R143 } \\
\hline $1993-98^{* \mathrm{c}}$ & late B & 11.9: & 9.9 & $\cdots$ \\
\hline $2003-07^{*}$ & late B & 11.6: & 9.6 & $\cdots$ \\
\hline $2008 \mathrm{Jul}^{*}$ & BA & 11.5: & 9.5 & $\cdots$ \\
\hline 2012 Sep & A abs & $?$ & $\cdots$ & $\cdots$ \\
\hline 2013 Mar & late B & $?$ & $\cdots$ & $\cdots$ \\
\hline 2014 Apr & A abs & $?$ & $\cdots$ & $\cdots$ \\
\hline $2016 \mathrm{Jul}^{*}$ & late B & 11.5 & 9.5 & $\cdots$ \\
\hline \multicolumn{5}{|l|}{ S Dor } \\
\hline $1999 \mathrm{Oct}^{\mathrm{c}}$ & $\mathrm{F}$ abs & 9.16 & 8.80 & (super)max? \\
\hline
\end{tabular}

Notes.

a $*$ high res.

b See Table 12 for classification criteria

c R127: Walborn (1977, 1982), Walborn et al. (2008); R71: Thackeray (1974); HDE 269582: Stahl (1986), Crowther \& Smith (1997); R143: Walborn (1997); S Dor: Massey (2000).

Table 12

Spectral Classification Criteria

\begin{tabular}{ll}
\hline \hline Sp.T. & Description \\
\hline Ofpe/WN9 & He II $\lambda 4686$, N III $\lambda \lambda 4634-40-42$ \\
Early B & Strong He I, N II $\lambda 3995 \& \lambda \lambda 4600$ multiplet, Si III $\lambda \lambda 4552-$ \\
& $68-75$ \\
Mid-B & Si II $\lambda \lambda 4128-30<$ He I $\lambda 4144$, Mg II $\lambda 4481<$ He I $\lambda 4471$ \\
B & Weak but definite He I, N II, Si III \\
& but main criteria N/A usually due to superimposed Fe II and \\
& other emission lines \\
Late B & Si II $\lambda \lambda 4128-30>$ He I $\lambda 4144$, Mg II $\lambda 4481>$ He I $\lambda 4471$ \\
BA & Possible very weak He I at low resolution \\
A & No He I except trace at high res; \\
& Strong Fe II absorption or emission, especially $\lambda \lambda 4173-79$, \\
& $4233,4924-5018-5169$ \\
F & Strong Fe II, Ba II, Ti II pure absorption spectra \\
\hline
\end{tabular}

\section{References}

Ardeberg, A., Brunet, J. P., Maurice, E., et al. 1972, A\&AS, 6, 249 (Erratum 7, 395)

Bagnulo, S., Jehin, E., Ledoux, C., et al. 2003, Msngr, 114, 10

Barbá, R. H., Gamen, R., Arias, J. I., et al. 2010, RMxAC, 38, 30

Bohannan, B., \& Walborn, N. R. 1989, PASP, 101, 520

Bonanos, A. Z., Massa, D. L., Sewiło, M., et al. 2009, AJ, 138, 1003

Butters, O. W., West, R. G., Anderson, D. R., et al. 2010, A\&A, 520, L10

Clayton, G. C., \& Martin, P. G. 1985, ApJ, 288, 558

Contreras, C., Hamuy, M., Phillips, M. M., et al. 2010, AJ, 139, 519

Crowther, P. A., \& Smith, L. J. 1997, A\&A, 320, 500

Dessart, L., Hillier, D. J., Gezari, S., et al. 2009, MNRAS, 394, 21

Feast, M. W., Thackeray, A. D., \& Wesselink, A. J. 1960, MNRAS, 121, 337

Gamen, R., Arias, J. I., Barbá, R. H., et al. 2012a, A\&A, 546, A92

Gamen, R., Barbá, R., Walborn, N., et al. 2009, IAUC, 9082, 1

Gamen, R., Walborn, N., Morrell, N., et al. 2012b, CBET, 3192, 1

Grindlay, J., Tang, S., Los, E., et al. 2012, IAUS, 285, 29

Groh, J. H., Meynet, G., \& Ekström, S. 2013, A\&A, 550, L7

Gummersbach, C. A., Zickgraf, F.-J., \& Wolf, B. 1995, A\&A, 302, 409

Heydari-Malayeri, M., Meynadier, F., \& Walborn, N. R. 2003, A\&A, 400, 923

Hodge, P. W., \& Wright, F. W. 1967, SAOP, 4699, 1

Howarth, I. D., \& Walborn, N. R. 2012, MNRAS, 426, 1867

Humphreys, R. M., Bond, H. E., Bedin, L. R., et al. 2011, ApJ, 743, 118

Hutsemékers, D., \& Van Drom, E. 1991, A\&A, 248, 141

Kaltcheva, N. T., \& Georgiev, L. N. 1992, MNRAS, 259, 166

Kaufer, A., Stahl, O., Tubbesing, S., et al. 1999, Msngr, 95, 8

Kotak, R., \& Vink, J. S. 2006, A\&A, 460, L5

Leitherer, C., Appenzeller, I., Klare, G., et al. 1985, A\&A, 153, 168

Mack, P., KanniahPadmanaban, S. Y., Kaitchuck, R., et al. 2010, in AAS Meeting 216 Abstracts, \#414.03

Maíz Apellániz, J., Sota, A., Walborn, N. R., et al. 2011, in Proc. IX Scientific Meeting of the Spanish Astronomical Society (SEA), Highlights of Spanish Astrophysics VI, ed. M. R. Zapatero Osorio et al., 467

Manfroid, J., Sterken, C., Bruch, A., et al. 1991, A\&AS, 87, 481

Massey, P. 2000, PASP, 112, 144

Massey, P., Morrell, N. I., Neugent, K. F., et al. 2012, ApJ, 748, 96

Massey, P., Neugent, K. F., Morrell, N., et al. 2014, ApJ, 788, 83

Mauerhan, J. C., Smith, N., Filippenko, A. V., et al. 2013a, MNRAS, 430, 1801

Mauerhan, J. C., Smith, N., Silverman, J. M., et al. 2013b, MNRAS, 431, 2599

Mehner, A., Baade, D., Rivinius, T., et al. 2013, A\&A, 555, A116

Mendoza, E. E. 1970, BOTT, 5, 269

Munari, U., Siviero, A., Bienaymé, O., et al. 2009, A\&A, 503, 511

Parker, J. Wm. 1993, AJ, 106, 560

Parker, J. Wm., Clayton, G. C., Winge, C., et al. 1993, ApJ, 409, 770

Pastorello, A., Botticella, M. T., Trundle, C., et al. 2010, MNRAS, 408, 181 Pojmanski, G. 1997, AcA, 47, 467

Prieto, J. L., Rest, A., Bianco, F. B., et al. 2014, ApJL, 787, L8

Prinja, R. K., \& Schild, H. 1991, A\&A, 249, 428

Rest, A., Prieto, J. L., Walborn, N. R., et al. 2012, Natur, 482, 375

Schild, H. 1987, A\&A, 173, 405

Schnurr, O., Moffat, A. F. J., St-Louis, N., et al. 2008, MNRAS, 389, 806

Shapley, H., \& Lindsay, E. M. 1963, IrAJ, 6, 74

Shore, S. N., \& Sanduleak, N. 1984, ApJS, 55, 1

Smith, L. J., Nota, A., Pasquali, A., et al. 1998, ApJ, 503, 278

Smith, N., Miller, A., Li, W., et al. 2010, AJ, 139, 1451

Sota, A., Maíz Apellániz, J., Morrell, N. I., et al. 2014, ApJS, 211, 10

Sota, A., Maíz Apellániz, J., Walborn, N. R., et al. 2011, ApJS, 193, 24

Spoon, H. W. W., de Koter, A., Sterken, C., et al. 1994, A\&AS, 106, 141

Stahl, O. 1986, A\&A, 164, 321 (Erratum 170, 197)

Szczygieł, D. M., Stanek, K. Z., Bonanos, A. Z., et al. 2010, AJ, 140, 14

Thackeray, A. D. 1974, MNRAS, 168, 221

van Genderen, A. M. 1979, A\&AS, 38, 151

van Genderen, A. M., \& Sterken, C. 1996, A\&A, 308, 763

van Leeuwen, F., van Genderen, A. M., \& Zegelaar, I. 1998, A\&AS, 128, 117

Walborn, N. R. 1977, ApJ, 215, 53

Walborn, N. R. 1982, ApJ, 256, 452

Walborn, N. R. 1991, IAUS, 143, 505

Walborn, N. R. 1997, ASPC, 120, 8 
Walborn, N. R., \& Blades, J. C. 1997, ApJS, 112, 457

Walborn, N. R., \& Fitzpatrick, E. L. 1990, PASP, 102, 379

Walborn, N. R., \& Fitzpatrick, E. L. 2000, PASP, 112, 50

Walborn, N. R., Gamen, R. C., Barbá, R. H., et al. 2014, ATel, 6295

Walborn, N. R., Gamen, R. C., Morrell, N. I., et al. 2012, in Massive Stars Workshop, Expanding Our Philosophy: Current Outbursts of Three LMC

LBV's, http://etacar.umn.edu/Workshop2012/Talks/Walborn.pdf Walborn, N. R., \& Howarth, I. D. 2000, PASP, 112, 1446
Walborn, N. R., Sana, H., Evans, C. J., et al. 2015, ApJ, 809, 109

Walborn, N. R., Stahl, O., Gamen, R. C., et al. 2008, ApJL, 683, L33

Weis, K. 2003, A\&A, 408, 205

Westerlund, B. 1961, Uppsala Astron Obs Ann, band 5, no 1

Wolf, B. 1975, A\&A, 41, 471

Wolf, B., Appenzeller, \& Stahl, O. 1981, A\&A, 103, 94

Wolf, B., \& Stahl, O. 1990, A\&A, 235, 340

Wolf, B., Stahl, O., Smolinski, J., et al. 1988, A\&AS, 74, 239 